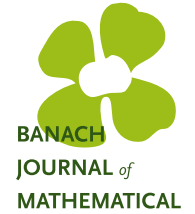

ANALYSIS

Banach J. Math. Anal. 10 (2016), no. 2, 338-384

http://dx.doi.org/10.1215/17358787-3495627

ISSN: $1735-8787$ (electronic)

http://projecteuclid.org/bjma

\title{
SQUARE FUNCTIONS AND SPECTRAL MULTIPLIERS FOR BESSEL OPERATORS IN UMD SPACES
}

\author{
JORGE J. BETANCOR, ${ }^{1}$ ALEJANDRO J. CASTRO,${ }^{2}$ and L. RODRÍGUEZ-MESA ${ }^{1 *}$ \\ Communicated by Q. Xu
}

\begin{abstract}
In this paper, we consider square functions (also called LittlewoodPaley $g$-functions) associated to Hankel convolutions acting on functions in the Bochner-Lebesgue space $L^{p}((0, \infty), \mathbb{B})$, where $\mathbb{B}$ is a UMD Banach space. As special cases, we study square functions defined by fractional derivatives of the Poisson semigroup for the Bessel operator $\Delta_{\lambda}=-x^{-\lambda} \frac{d}{d x} x^{2 \lambda} \frac{d}{d x} x^{-\lambda}$, $\lambda>0$. We characterize the UMD property for a Banach space $\mathbb{B}$ by using $L^{p}((0, \infty), \mathbb{B})$-boundedness properties of $g$-functions defined by Bessel-Poisson semigroups. As a by-product, we prove that the fact that the imaginary power $\Delta_{\lambda}^{i \omega}, \omega \in \mathbb{R} \backslash\{0\}$, of the Bessel operator $\Delta_{\lambda}$ is bounded in $L^{p}((0, \infty), \mathbb{B})$, $1<p<\infty$, characterizes the UMD property for the Banach space $\mathbb{B}$. As applications of our results for square functions, we establish the boundedness in $L^{p}((0, \infty), \mathbb{B})$ of spectral multipliers $m\left(\Delta_{\lambda}\right)$ of Bessel operators defined by functions $m$ which are holomorphic in sectors $\Sigma_{\vartheta}$.
\end{abstract}

\section{Introduction}

Square functions (also called Littlewood-Paley $g$-functions) were considered in the works of Littlewood, Paley, Zygmund, and Marcinkiewicz during the decade of the 1930s (see [34], [55]). These functions were introduced to get new equivalent norms, for example, in $L^{p}$-spaces. By using these new equivalent norms the boundedness of some operators (e.g., multipliers) can be established.

Copyright 2016 by the Tusi Mathematical Research Group.

Received Apr. 10, 2015; Accepted Jul. 9, 2015.

${ }^{*}$ Corresponding author.

2010 Mathematics Subject Classification. Primary 42A25; Secondary 42B20, 43A15, 46B20, 46E40, 47D03.

Keywords. UMD space, square function, spectral multiplier, Bessel operator, $\gamma$-radonifying operator. 


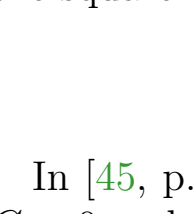

ANALYSIS

Banach J. Math. Anal. 10 (2016), no. 2, 338-384

http://dx.doi.org/10.1215/17358787-3495627

ISSN: $1735-8787$ (electronic)

http://projecteuclid.org/bjma

\title{
SQUARE FUNCTIONS AND SPECTRAL MULTIPLIERS FOR BESSEL OPERATORS IN UMD SPACES
}

\author{
JORGE J. BETANCOR, ${ }^{1}$ ALEJANDRO J. CASTRO,${ }^{2}$ and L. RODRÍGUEZ-MESA ${ }^{1 *}$ \\ Communicated by Q. Xu
}

\begin{abstract}
In this paper, we consider square functions (also called LittlewoodPaley $g$-functions) associated to Hankel convolutions acting on functions in the Bochner-Lebesgue space $L^{p}((0, \infty), \mathbb{B})$, where $\mathbb{B}$ is a UMD Banach space. As special cases, we study square functions defined by fractional derivatives of the Poisson semigroup for the Bessel operator $\Delta_{\lambda}=-x^{-\lambda} \frac{d}{d x} x^{2 \lambda} \frac{d}{d x} x^{-\lambda}$, $\lambda>0$. We characterize the UMD property for a Banach space $\mathbb{B}$ by using $L^{p}((0, \infty), \mathbb{B})$-boundedness properties of $g$-functions defined by Bessel-Poisson semigroups. As a by-product, we prove that the fact that the imaginary power $\Delta_{\lambda}^{i \omega}, \omega \in \mathbb{R} \backslash\{0\}$, of the Bessel operator $\Delta_{\lambda}$ is bounded in $L^{p}((0, \infty), \mathbb{B})$, $1<p<\infty$, characterizes the UMD property for the Banach space $\mathbb{B}$. As applications of our results for square functions, we establish the boundedness in $L^{p}((0, \infty), \mathbb{B})$ of spectral multipliers $m\left(\Delta_{\lambda}\right)$ of Bessel operators defined by functions $m$ which are holomorphic in sectors $\Sigma_{\vartheta}$.
\end{abstract}

\section{Introduction}

Square functions (also called Littlewood-Paley $g$-functions) were considered in the works of Littlewood, Paley, Zygmund, and Marcinkiewicz during the decade of the 1930s (see [34], [55]). These functions were introduced to get new equivalent norms, for example, in $L^{p}$-spaces. By using these new equivalent norms the boundedness of some operators (e.g., multipliers) can be established.

Copyright 2016 by the Tusi Mathematical Research Group.

Received Apr. 10, 2015; Accepted Jul. 9, 2015.

${ }^{*}$ Corresponding author.

2010 Mathematics Subject Classification. Primary 42A25; Secondary 42B20, 43A15, 46B20, 46E40, 47D03.

Keywords. UMD space, square function, spectral multiplier, Bessel operator, $\gamma$-radonifying operator. 


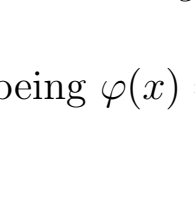

ANALYSIS

Banach J. Math. Anal. 10 (2016), no. 2, 338-384

http://dx.doi.org/10.1215/17358787-3495627

ISSN: $1735-8787$ (electronic)

http://projecteuclid.org/bjma

\title{
SQUARE FUNCTIONS AND SPECTRAL MULTIPLIERS FOR BESSEL OPERATORS IN UMD SPACES
}

\author{
JORGE J. BETANCOR, ${ }^{1}$ ALEJANDRO J. CASTRO,${ }^{2}$ and L. RODRÍGUEZ-MESA ${ }^{1 *}$ \\ Communicated by Q. Xu
}

\begin{abstract}
In this paper, we consider square functions (also called LittlewoodPaley $g$-functions) associated to Hankel convolutions acting on functions in the Bochner-Lebesgue space $L^{p}((0, \infty), \mathbb{B})$, where $\mathbb{B}$ is a UMD Banach space. As special cases, we study square functions defined by fractional derivatives of the Poisson semigroup for the Bessel operator $\Delta_{\lambda}=-x^{-\lambda} \frac{d}{d x} x^{2 \lambda} \frac{d}{d x} x^{-\lambda}$, $\lambda>0$. We characterize the UMD property for a Banach space $\mathbb{B}$ by using $L^{p}((0, \infty), \mathbb{B})$-boundedness properties of $g$-functions defined by Bessel-Poisson semigroups. As a by-product, we prove that the fact that the imaginary power $\Delta_{\lambda}^{i \omega}, \omega \in \mathbb{R} \backslash\{0\}$, of the Bessel operator $\Delta_{\lambda}$ is bounded in $L^{p}((0, \infty), \mathbb{B})$, $1<p<\infty$, characterizes the UMD property for the Banach space $\mathbb{B}$. As applications of our results for square functions, we establish the boundedness in $L^{p}((0, \infty), \mathbb{B})$ of spectral multipliers $m\left(\Delta_{\lambda}\right)$ of Bessel operators defined by functions $m$ which are holomorphic in sectors $\Sigma_{\vartheta}$.
\end{abstract}

\section{Introduction}

Square functions (also called Littlewood-Paley $g$-functions) were considered in the works of Littlewood, Paley, Zygmund, and Marcinkiewicz during the decade of the 1930s (see [34], [55]). These functions were introduced to get new equivalent norms, for example, in $L^{p}$-spaces. By using these new equivalent norms the boundedness of some operators (e.g., multipliers) can be established.

Copyright 2016 by the Tusi Mathematical Research Group.

Received Apr. 10, 2015; Accepted Jul. 9, 2015.

${ }^{*}$ Corresponding author.

2010 Mathematics Subject Classification. Primary 42A25; Secondary 42B20, 43A15, 46B20, 46E40, 47D03.

Keywords. UMD space, square function, spectral multiplier, Bessel operator, $\gamma$-radonifying operator. 


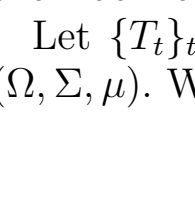

ANALYSIS

Banach J. Math. Anal. 10 (2016), no. 2, 338-384

http://dx.doi.org/10.1215/17358787-3495627

ISSN: $1735-8787$ (electronic)

http://projecteuclid.org/bjma

\title{
SQUARE FUNCTIONS AND SPECTRAL MULTIPLIERS FOR BESSEL OPERATORS IN UMD SPACES
}

\author{
JORGE J. BETANCOR, ${ }^{1}$ ALEJANDRO J. CASTRO,${ }^{2}$ and L. RODRÍGUEZ-MESA ${ }^{1 *}$ \\ Communicated by Q. Xu
}

\begin{abstract}
In this paper, we consider square functions (also called LittlewoodPaley $g$-functions) associated to Hankel convolutions acting on functions in the Bochner-Lebesgue space $L^{p}((0, \infty), \mathbb{B})$, where $\mathbb{B}$ is a UMD Banach space. As special cases, we study square functions defined by fractional derivatives of the Poisson semigroup for the Bessel operator $\Delta_{\lambda}=-x^{-\lambda} \frac{d}{d x} x^{2 \lambda} \frac{d}{d x} x^{-\lambda}$, $\lambda>0$. We characterize the UMD property for a Banach space $\mathbb{B}$ by using $L^{p}((0, \infty), \mathbb{B})$-boundedness properties of $g$-functions defined by Bessel-Poisson semigroups. As a by-product, we prove that the fact that the imaginary power $\Delta_{\lambda}^{i \omega}, \omega \in \mathbb{R} \backslash\{0\}$, of the Bessel operator $\Delta_{\lambda}$ is bounded in $L^{p}((0, \infty), \mathbb{B})$, $1<p<\infty$, characterizes the UMD property for the Banach space $\mathbb{B}$. As applications of our results for square functions, we establish the boundedness in $L^{p}((0, \infty), \mathbb{B})$ of spectral multipliers $m\left(\Delta_{\lambda}\right)$ of Bessel operators defined by functions $m$ which are holomorphic in sectors $\Sigma_{\vartheta}$.
\end{abstract}

\section{Introduction}

Square functions (also called Littlewood-Paley $g$-functions) were considered in the works of Littlewood, Paley, Zygmund, and Marcinkiewicz during the decade of the 1930s (see [34], [55]). These functions were introduced to get new equivalent norms, for example, in $L^{p}$-spaces. By using these new equivalent norms the boundedness of some operators (e.g., multipliers) can be established.

Copyright 2016 by the Tusi Mathematical Research Group.

Received Apr. 10, 2015; Accepted Jul. 9, 2015.

${ }^{*}$ Corresponding author.

2010 Mathematics Subject Classification. Primary 42A25; Secondary 42B20, 43A15, 46B20, 46E40, 47D03.

Keywords. UMD space, square function, spectral multiplier, Bessel operator, $\gamma$-radonifying operator. 


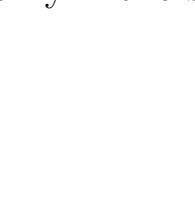

ANALYSIS

Banach J. Math. Anal. 10 (2016), no. 2, 338-384

http://dx.doi.org/10.1215/17358787-3495627

ISSN: $1735-8787$ (electronic)

http://projecteuclid.org/bjma

\title{
SQUARE FUNCTIONS AND SPECTRAL MULTIPLIERS FOR BESSEL OPERATORS IN UMD SPACES
}

\author{
JORGE J. BETANCOR, ${ }^{1}$ ALEJANDRO J. CASTRO,${ }^{2}$ and L. RODRÍGUEZ-MESA ${ }^{1 *}$ \\ Communicated by Q. Xu
}

\begin{abstract}
In this paper, we consider square functions (also called LittlewoodPaley $g$-functions) associated to Hankel convolutions acting on functions in the Bochner-Lebesgue space $L^{p}((0, \infty), \mathbb{B})$, where $\mathbb{B}$ is a UMD Banach space. As special cases, we study square functions defined by fractional derivatives of the Poisson semigroup for the Bessel operator $\Delta_{\lambda}=-x^{-\lambda} \frac{d}{d x} x^{2 \lambda} \frac{d}{d x} x^{-\lambda}$, $\lambda>0$. We characterize the UMD property for a Banach space $\mathbb{B}$ by using $L^{p}((0, \infty), \mathbb{B})$-boundedness properties of $g$-functions defined by Bessel-Poisson semigroups. As a by-product, we prove that the fact that the imaginary power $\Delta_{\lambda}^{i \omega}, \omega \in \mathbb{R} \backslash\{0\}$, of the Bessel operator $\Delta_{\lambda}$ is bounded in $L^{p}((0, \infty), \mathbb{B})$, $1<p<\infty$, characterizes the UMD property for the Banach space $\mathbb{B}$. As applications of our results for square functions, we establish the boundedness in $L^{p}((0, \infty), \mathbb{B})$ of spectral multipliers $m\left(\Delta_{\lambda}\right)$ of Bessel operators defined by functions $m$ which are holomorphic in sectors $\Sigma_{\vartheta}$.
\end{abstract}

\section{Introduction}

Square functions (also called Littlewood-Paley $g$-functions) were considered in the works of Littlewood, Paley, Zygmund, and Marcinkiewicz during the decade of the 1930s (see [34], [55]). These functions were introduced to get new equivalent norms, for example, in $L^{p}$-spaces. By using these new equivalent norms the boundedness of some operators (e.g., multipliers) can be established.

Copyright 2016 by the Tusi Mathematical Research Group.

Received Apr. 10, 2015; Accepted Jul. 9, 2015.

${ }^{*}$ Corresponding author.

2010 Mathematics Subject Classification. Primary 42A25; Secondary 42B20, 43A15, 46B20, 46E40, 47D03.

Keywords. UMD space, square function, spectral multiplier, Bessel operator, $\gamma$-radonifying operator. 


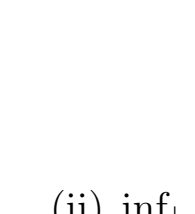

ANALYSIS

Banach J. Math. Anal. 10 (2016), no. 2, 338-384

http://dx.doi.org/10.1215/17358787-3495627

ISSN: $1735-8787$ (electronic)

http://projecteuclid.org/bjma

\title{
SQUARE FUNCTIONS AND SPECTRAL MULTIPLIERS FOR BESSEL OPERATORS IN UMD SPACES
}

\author{
JORGE J. BETANCOR, ${ }^{1}$ ALEJANDRO J. CASTRO,${ }^{2}$ and L. RODRÍGUEZ-MESA ${ }^{1 *}$ \\ Communicated by Q. Xu
}

\begin{abstract}
In this paper, we consider square functions (also called LittlewoodPaley $g$-functions) associated to Hankel convolutions acting on functions in the Bochner-Lebesgue space $L^{p}((0, \infty), \mathbb{B})$, where $\mathbb{B}$ is a UMD Banach space. As special cases, we study square functions defined by fractional derivatives of the Poisson semigroup for the Bessel operator $\Delta_{\lambda}=-x^{-\lambda} \frac{d}{d x} x^{2 \lambda} \frac{d}{d x} x^{-\lambda}$, $\lambda>0$. We characterize the UMD property for a Banach space $\mathbb{B}$ by using $L^{p}((0, \infty), \mathbb{B})$-boundedness properties of $g$-functions defined by Bessel-Poisson semigroups. As a by-product, we prove that the fact that the imaginary power $\Delta_{\lambda}^{i \omega}, \omega \in \mathbb{R} \backslash\{0\}$, of the Bessel operator $\Delta_{\lambda}$ is bounded in $L^{p}((0, \infty), \mathbb{B})$, $1<p<\infty$, characterizes the UMD property for the Banach space $\mathbb{B}$. As applications of our results for square functions, we establish the boundedness in $L^{p}((0, \infty), \mathbb{B})$ of spectral multipliers $m\left(\Delta_{\lambda}\right)$ of Bessel operators defined by functions $m$ which are holomorphic in sectors $\Sigma_{\vartheta}$.
\end{abstract}

\section{Introduction}

Square functions (also called Littlewood-Paley $g$-functions) were considered in the works of Littlewood, Paley, Zygmund, and Marcinkiewicz during the decade of the 1930s (see [34], [55]). These functions were introduced to get new equivalent norms, for example, in $L^{p}$-spaces. By using these new equivalent norms the boundedness of some operators (e.g., multipliers) can be established.

Copyright 2016 by the Tusi Mathematical Research Group.

Received Apr. 10, 2015; Accepted Jul. 9, 2015.

${ }^{*}$ Corresponding author.

2010 Mathematics Subject Classification. Primary 42A25; Secondary 42B20, 43A15, 46B20, 46E40, 47D03.

Keywords. UMD space, square function, spectral multiplier, Bessel operator, $\gamma$-radonifying operator. 


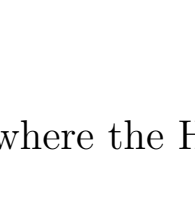

ANALYSIS

Banach J. Math. Anal. 10 (2016), no. 2, 338-384

http://dx.doi.org/10.1215/17358787-3495627

ISSN: $1735-8787$ (electronic)

http://projecteuclid.org/bjma

\title{
SQUARE FUNCTIONS AND SPECTRAL MULTIPLIERS FOR BESSEL OPERATORS IN UMD SPACES
}

\author{
JORGE J. BETANCOR, ${ }^{1}$ ALEJANDRO J. CASTRO,${ }^{2}$ and L. RODRÍGUEZ-MESA ${ }^{1 *}$ \\ Communicated by Q. Xu
}

\begin{abstract}
In this paper, we consider square functions (also called LittlewoodPaley $g$-functions) associated to Hankel convolutions acting on functions in the Bochner-Lebesgue space $L^{p}((0, \infty), \mathbb{B})$, where $\mathbb{B}$ is a UMD Banach space. As special cases, we study square functions defined by fractional derivatives of the Poisson semigroup for the Bessel operator $\Delta_{\lambda}=-x^{-\lambda} \frac{d}{d x} x^{2 \lambda} \frac{d}{d x} x^{-\lambda}$, $\lambda>0$. We characterize the UMD property for a Banach space $\mathbb{B}$ by using $L^{p}((0, \infty), \mathbb{B})$-boundedness properties of $g$-functions defined by Bessel-Poisson semigroups. As a by-product, we prove that the fact that the imaginary power $\Delta_{\lambda}^{i \omega}, \omega \in \mathbb{R} \backslash\{0\}$, of the Bessel operator $\Delta_{\lambda}$ is bounded in $L^{p}((0, \infty), \mathbb{B})$, $1<p<\infty$, characterizes the UMD property for the Banach space $\mathbb{B}$. As applications of our results for square functions, we establish the boundedness in $L^{p}((0, \infty), \mathbb{B})$ of spectral multipliers $m\left(\Delta_{\lambda}\right)$ of Bessel operators defined by functions $m$ which are holomorphic in sectors $\Sigma_{\vartheta}$.
\end{abstract}

\section{Introduction}

Square functions (also called Littlewood-Paley $g$-functions) were considered in the works of Littlewood, Paley, Zygmund, and Marcinkiewicz during the decade of the 1930s (see [34], [55]). These functions were introduced to get new equivalent norms, for example, in $L^{p}$-spaces. By using these new equivalent norms the boundedness of some operators (e.g., multipliers) can be established.

Copyright 2016 by the Tusi Mathematical Research Group.

Received Apr. 10, 2015; Accepted Jul. 9, 2015.

${ }^{*}$ Corresponding author.

2010 Mathematics Subject Classification. Primary 42A25; Secondary 42B20, 43A15, 46B20, 46E40, 47D03.

Keywords. UMD space, square function, spectral multiplier, Bessel operator, $\gamma$-radonifying operator. 


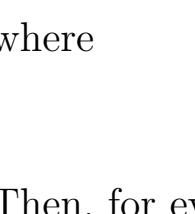

ANALYSIS

Banach J. Math. Anal. 10 (2016), no. 2, 338-384

http://dx.doi.org/10.1215/17358787-3495627

ISSN: $1735-8787$ (electronic)

http://projecteuclid.org/bjma

\title{
SQUARE FUNCTIONS AND SPECTRAL MULTIPLIERS FOR BESSEL OPERATORS IN UMD SPACES
}

\author{
JORGE J. BETANCOR, ${ }^{1}$ ALEJANDRO J. CASTRO,${ }^{2}$ and L. RODRÍGUEZ-MESA ${ }^{1 *}$ \\ Communicated by Q. Xu
}

\begin{abstract}
In this paper, we consider square functions (also called LittlewoodPaley $g$-functions) associated to Hankel convolutions acting on functions in the Bochner-Lebesgue space $L^{p}((0, \infty), \mathbb{B})$, where $\mathbb{B}$ is a UMD Banach space. As special cases, we study square functions defined by fractional derivatives of the Poisson semigroup for the Bessel operator $\Delta_{\lambda}=-x^{-\lambda} \frac{d}{d x} x^{2 \lambda} \frac{d}{d x} x^{-\lambda}$, $\lambda>0$. We characterize the UMD property for a Banach space $\mathbb{B}$ by using $L^{p}((0, \infty), \mathbb{B})$-boundedness properties of $g$-functions defined by Bessel-Poisson semigroups. As a by-product, we prove that the fact that the imaginary power $\Delta_{\lambda}^{i \omega}, \omega \in \mathbb{R} \backslash\{0\}$, of the Bessel operator $\Delta_{\lambda}$ is bounded in $L^{p}((0, \infty), \mathbb{B})$, $1<p<\infty$, characterizes the UMD property for the Banach space $\mathbb{B}$. As applications of our results for square functions, we establish the boundedness in $L^{p}((0, \infty), \mathbb{B})$ of spectral multipliers $m\left(\Delta_{\lambda}\right)$ of Bessel operators defined by functions $m$ which are holomorphic in sectors $\Sigma_{\vartheta}$.
\end{abstract}

\section{Introduction}

Square functions (also called Littlewood-Paley $g$-functions) were considered in the works of Littlewood, Paley, Zygmund, and Marcinkiewicz during the decade of the 1930s (see [34], [55]). These functions were introduced to get new equivalent norms, for example, in $L^{p}$-spaces. By using these new equivalent norms the boundedness of some operators (e.g., multipliers) can be established.

Copyright 2016 by the Tusi Mathematical Research Group.

Received Apr. 10, 2015; Accepted Jul. 9, 2015.

${ }^{*}$ Corresponding author.

2010 Mathematics Subject Classification. Primary 42A25; Secondary 42B20, 43A15, 46B20, 46E40, 47D03.

Keywords. UMD space, square function, spectral multiplier, Bessel operator, $\gamma$-radonifying operator. 


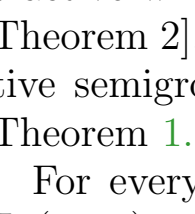

ANALYSIS

Banach J. Math. Anal. 10 (2016), no. 2, 338-384

http://dx.doi.org/10.1215/17358787-3495627

ISSN: $1735-8787$ (electronic)

http://projecteuclid.org/bjma

\title{
SQUARE FUNCTIONS AND SPECTRAL MULTIPLIERS FOR BESSEL OPERATORS IN UMD SPACES
}

\author{
JORGE J. BETANCOR, ${ }^{1}$ ALEJANDRO J. CASTRO,${ }^{2}$ and L. RODRÍGUEZ-MESA ${ }^{1 *}$ \\ Communicated by Q. Xu
}

\begin{abstract}
In this paper, we consider square functions (also called LittlewoodPaley $g$-functions) associated to Hankel convolutions acting on functions in the Bochner-Lebesgue space $L^{p}((0, \infty), \mathbb{B})$, where $\mathbb{B}$ is a UMD Banach space. As special cases, we study square functions defined by fractional derivatives of the Poisson semigroup for the Bessel operator $\Delta_{\lambda}=-x^{-\lambda} \frac{d}{d x} x^{2 \lambda} \frac{d}{d x} x^{-\lambda}$, $\lambda>0$. We characterize the UMD property for a Banach space $\mathbb{B}$ by using $L^{p}((0, \infty), \mathbb{B})$-boundedness properties of $g$-functions defined by Bessel-Poisson semigroups. As a by-product, we prove that the fact that the imaginary power $\Delta_{\lambda}^{i \omega}, \omega \in \mathbb{R} \backslash\{0\}$, of the Bessel operator $\Delta_{\lambda}$ is bounded in $L^{p}((0, \infty), \mathbb{B})$, $1<p<\infty$, characterizes the UMD property for the Banach space $\mathbb{B}$. As applications of our results for square functions, we establish the boundedness in $L^{p}((0, \infty), \mathbb{B})$ of spectral multipliers $m\left(\Delta_{\lambda}\right)$ of Bessel operators defined by functions $m$ which are holomorphic in sectors $\Sigma_{\vartheta}$.
\end{abstract}

\section{Introduction}

Square functions (also called Littlewood-Paley $g$-functions) were considered in the works of Littlewood, Paley, Zygmund, and Marcinkiewicz during the decade of the 1930s (see [34], [55]). These functions were introduced to get new equivalent norms, for example, in $L^{p}$-spaces. By using these new equivalent norms the boundedness of some operators (e.g., multipliers) can be established.

Copyright 2016 by the Tusi Mathematical Research Group.

Received Apr. 10, 2015; Accepted Jul. 9, 2015.

${ }^{*}$ Corresponding author.

2010 Mathematics Subject Classification. Primary 42A25; Secondary 42B20, 43A15, 46B20, 46E40, 47D03.

Keywords. UMD space, square function, spectral multiplier, Bessel operator, $\gamma$-radonifying operator. 


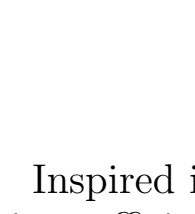

ANALYSIS

Banach J. Math. Anal. 10 (2016), no. 2, 338-384

http://dx.doi.org/10.1215/17358787-3495627

ISSN: $1735-8787$ (electronic)

http://projecteuclid.org/bjma

\title{
SQUARE FUNCTIONS AND SPECTRAL MULTIPLIERS FOR BESSEL OPERATORS IN UMD SPACES
}

\author{
JORGE J. BETANCOR, ${ }^{1}$ ALEJANDRO J. CASTRO,${ }^{2}$ and L. RODRÍGUEZ-MESA ${ }^{1 *}$ \\ Communicated by Q. Xu
}

\begin{abstract}
In this paper, we consider square functions (also called LittlewoodPaley $g$-functions) associated to Hankel convolutions acting on functions in the Bochner-Lebesgue space $L^{p}((0, \infty), \mathbb{B})$, where $\mathbb{B}$ is a UMD Banach space. As special cases, we study square functions defined by fractional derivatives of the Poisson semigroup for the Bessel operator $\Delta_{\lambda}=-x^{-\lambda} \frac{d}{d x} x^{2 \lambda} \frac{d}{d x} x^{-\lambda}$, $\lambda>0$. We characterize the UMD property for a Banach space $\mathbb{B}$ by using $L^{p}((0, \infty), \mathbb{B})$-boundedness properties of $g$-functions defined by Bessel-Poisson semigroups. As a by-product, we prove that the fact that the imaginary power $\Delta_{\lambda}^{i \omega}, \omega \in \mathbb{R} \backslash\{0\}$, of the Bessel operator $\Delta_{\lambda}$ is bounded in $L^{p}((0, \infty), \mathbb{B})$, $1<p<\infty$, characterizes the UMD property for the Banach space $\mathbb{B}$. As applications of our results for square functions, we establish the boundedness in $L^{p}((0, \infty), \mathbb{B})$ of spectral multipliers $m\left(\Delta_{\lambda}\right)$ of Bessel operators defined by functions $m$ which are holomorphic in sectors $\Sigma_{\vartheta}$.
\end{abstract}

\section{Introduction}

Square functions (also called Littlewood-Paley $g$-functions) were considered in the works of Littlewood, Paley, Zygmund, and Marcinkiewicz during the decade of the 1930s (see [34], [55]). These functions were introduced to get new equivalent norms, for example, in $L^{p}$-spaces. By using these new equivalent norms the boundedness of some operators (e.g., multipliers) can be established.

Copyright 2016 by the Tusi Mathematical Research Group.

Received Apr. 10, 2015; Accepted Jul. 9, 2015.

${ }^{*}$ Corresponding author.

2010 Mathematics Subject Classification. Primary 42A25; Secondary 42B20, 43A15, 46B20, 46E40, 47D03.

Keywords. UMD space, square function, spectral multiplier, Bessel operator, $\gamma$-radonifying operator. 


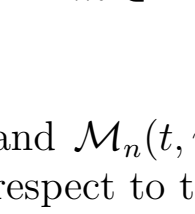

ANALYSIS

Banach J. Math. Anal. 10 (2016), no. 2, 338-384

http://dx.doi.org/10.1215/17358787-3495627

ISSN: $1735-8787$ (electronic)

http://projecteuclid.org/bjma

\title{
SQUARE FUNCTIONS AND SPECTRAL MULTIPLIERS FOR BESSEL OPERATORS IN UMD SPACES
}

\author{
JORGE J. BETANCOR, ${ }^{1}$ ALEJANDRO J. CASTRO,${ }^{2}$ and L. RODRÍGUEZ-MESA ${ }^{1 *}$ \\ Communicated by Q. Xu
}

\begin{abstract}
In this paper, we consider square functions (also called LittlewoodPaley $g$-functions) associated to Hankel convolutions acting on functions in the Bochner-Lebesgue space $L^{p}((0, \infty), \mathbb{B})$, where $\mathbb{B}$ is a UMD Banach space. As special cases, we study square functions defined by fractional derivatives of the Poisson semigroup for the Bessel operator $\Delta_{\lambda}=-x^{-\lambda} \frac{d}{d x} x^{2 \lambda} \frac{d}{d x} x^{-\lambda}$, $\lambda>0$. We characterize the UMD property for a Banach space $\mathbb{B}$ by using $L^{p}((0, \infty), \mathbb{B})$-boundedness properties of $g$-functions defined by Bessel-Poisson semigroups. As a by-product, we prove that the fact that the imaginary power $\Delta_{\lambda}^{i \omega}, \omega \in \mathbb{R} \backslash\{0\}$, of the Bessel operator $\Delta_{\lambda}$ is bounded in $L^{p}((0, \infty), \mathbb{B})$, $1<p<\infty$, characterizes the UMD property for the Banach space $\mathbb{B}$. As applications of our results for square functions, we establish the boundedness in $L^{p}((0, \infty), \mathbb{B})$ of spectral multipliers $m\left(\Delta_{\lambda}\right)$ of Bessel operators defined by functions $m$ which are holomorphic in sectors $\Sigma_{\vartheta}$.
\end{abstract}

\section{Introduction}

Square functions (also called Littlewood-Paley $g$-functions) were considered in the works of Littlewood, Paley, Zygmund, and Marcinkiewicz during the decade of the 1930s (see [34], [55]). These functions were introduced to get new equivalent norms, for example, in $L^{p}$-spaces. By using these new equivalent norms the boundedness of some operators (e.g., multipliers) can be established.

Copyright 2016 by the Tusi Mathematical Research Group.

Received Apr. 10, 2015; Accepted Jul. 9, 2015.

${ }^{*}$ Corresponding author.

2010 Mathematics Subject Classification. Primary 42A25; Secondary 42B20, 43A15, 46B20, 46E40, 47D03.

Keywords. UMD space, square function, spectral multiplier, Bessel operator, $\gamma$-radonifying operator. 


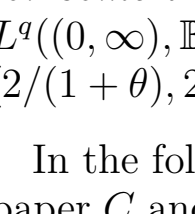

ANALYSIS

Banach J. Math. Anal. 10 (2016), no. 2, 338-384

http://dx.doi.org/10.1215/17358787-3495627

ISSN: $1735-8787$ (electronic)

http://projecteuclid.org/bjma

\title{
SQUARE FUNCTIONS AND SPECTRAL MULTIPLIERS FOR BESSEL OPERATORS IN UMD SPACES
}

\author{
JORGE J. BETANCOR, ${ }^{1}$ ALEJANDRO J. CASTRO,${ }^{2}$ and L. RODRÍGUEZ-MESA ${ }^{1 *}$ \\ Communicated by Q. Xu
}

\begin{abstract}
In this paper, we consider square functions (also called LittlewoodPaley $g$-functions) associated to Hankel convolutions acting on functions in the Bochner-Lebesgue space $L^{p}((0, \infty), \mathbb{B})$, where $\mathbb{B}$ is a UMD Banach space. As special cases, we study square functions defined by fractional derivatives of the Poisson semigroup for the Bessel operator $\Delta_{\lambda}=-x^{-\lambda} \frac{d}{d x} x^{2 \lambda} \frac{d}{d x} x^{-\lambda}$, $\lambda>0$. We characterize the UMD property for a Banach space $\mathbb{B}$ by using $L^{p}((0, \infty), \mathbb{B})$-boundedness properties of $g$-functions defined by Bessel-Poisson semigroups. As a by-product, we prove that the fact that the imaginary power $\Delta_{\lambda}^{i \omega}, \omega \in \mathbb{R} \backslash\{0\}$, of the Bessel operator $\Delta_{\lambda}$ is bounded in $L^{p}((0, \infty), \mathbb{B})$, $1<p<\infty$, characterizes the UMD property for the Banach space $\mathbb{B}$. As applications of our results for square functions, we establish the boundedness in $L^{p}((0, \infty), \mathbb{B})$ of spectral multipliers $m\left(\Delta_{\lambda}\right)$ of Bessel operators defined by functions $m$ which are holomorphic in sectors $\Sigma_{\vartheta}$.
\end{abstract}

\section{Introduction}

Square functions (also called Littlewood-Paley $g$-functions) were considered in the works of Littlewood, Paley, Zygmund, and Marcinkiewicz during the decade of the 1930s (see [34], [55]). These functions were introduced to get new equivalent norms, for example, in $L^{p}$-spaces. By using these new equivalent norms the boundedness of some operators (e.g., multipliers) can be established.

Copyright 2016 by the Tusi Mathematical Research Group.

Received Apr. 10, 2015; Accepted Jul. 9, 2015.

${ }^{*}$ Corresponding author.

2010 Mathematics Subject Classification. Primary 42A25; Secondary 42B20, 43A15, 46B20, 46E40, 47D03.

Keywords. UMD space, square function, spectral multiplier, Bessel operator, $\gamma$-radonifying operator. 


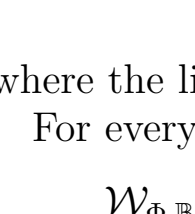

ANALYSIS

Banach J. Math. Anal. 10 (2016), no. 2, 338-384

http://dx.doi.org/10.1215/17358787-3495627

ISSN: $1735-8787$ (electronic)

http://projecteuclid.org/bjma

\title{
SQUARE FUNCTIONS AND SPECTRAL MULTIPLIERS FOR BESSEL OPERATORS IN UMD SPACES
}

\author{
JORGE J. BETANCOR, ${ }^{1}$ ALEJANDRO J. CASTRO,${ }^{2}$ and L. RODRÍGUEZ-MESA ${ }^{1 *}$ \\ Communicated by Q. Xu
}

\begin{abstract}
In this paper, we consider square functions (also called LittlewoodPaley $g$-functions) associated to Hankel convolutions acting on functions in the Bochner-Lebesgue space $L^{p}((0, \infty), \mathbb{B})$, where $\mathbb{B}$ is a UMD Banach space. As special cases, we study square functions defined by fractional derivatives of the Poisson semigroup for the Bessel operator $\Delta_{\lambda}=-x^{-\lambda} \frac{d}{d x} x^{2 \lambda} \frac{d}{d x} x^{-\lambda}$, $\lambda>0$. We characterize the UMD property for a Banach space $\mathbb{B}$ by using $L^{p}((0, \infty), \mathbb{B})$-boundedness properties of $g$-functions defined by Bessel-Poisson semigroups. As a by-product, we prove that the fact that the imaginary power $\Delta_{\lambda}^{i \omega}, \omega \in \mathbb{R} \backslash\{0\}$, of the Bessel operator $\Delta_{\lambda}$ is bounded in $L^{p}((0, \infty), \mathbb{B})$, $1<p<\infty$, characterizes the UMD property for the Banach space $\mathbb{B}$. As applications of our results for square functions, we establish the boundedness in $L^{p}((0, \infty), \mathbb{B})$ of spectral multipliers $m\left(\Delta_{\lambda}\right)$ of Bessel operators defined by functions $m$ which are holomorphic in sectors $\Sigma_{\vartheta}$.
\end{abstract}

\section{Introduction}

Square functions (also called Littlewood-Paley $g$-functions) were considered in the works of Littlewood, Paley, Zygmund, and Marcinkiewicz during the decade of the 1930s (see [34], [55]). These functions were introduced to get new equivalent norms, for example, in $L^{p}$-spaces. By using these new equivalent norms the boundedness of some operators (e.g., multipliers) can be established.

Copyright 2016 by the Tusi Mathematical Research Group.

Received Apr. 10, 2015; Accepted Jul. 9, 2015.

${ }^{*}$ Corresponding author.

2010 Mathematics Subject Classification. Primary 42A25; Secondary 42B20, 43A15, 46B20, 46E40, 47D03.

Keywords. UMD space, square function, spectral multiplier, Bessel operator, $\gamma$-radonifying operator. 


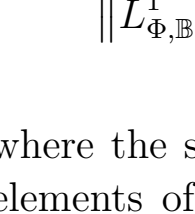

ANALYSIS

Banach J. Math. Anal. 10 (2016), no. 2, 338-384

http://dx.doi.org/10.1215/17358787-3495627

ISSN: $1735-8787$ (electronic)

http://projecteuclid.org/bjma

\title{
SQUARE FUNCTIONS AND SPECTRAL MULTIPLIERS FOR BESSEL OPERATORS IN UMD SPACES
}

\author{
JORGE J. BETANCOR, ${ }^{1}$ ALEJANDRO J. CASTRO,${ }^{2}$ and L. RODRÍGUEZ-MESA ${ }^{1 *}$ \\ Communicated by Q. Xu
}

\begin{abstract}
In this paper, we consider square functions (also called LittlewoodPaley $g$-functions) associated to Hankel convolutions acting on functions in the Bochner-Lebesgue space $L^{p}((0, \infty), \mathbb{B})$, where $\mathbb{B}$ is a UMD Banach space. As special cases, we study square functions defined by fractional derivatives of the Poisson semigroup for the Bessel operator $\Delta_{\lambda}=-x^{-\lambda} \frac{d}{d x} x^{2 \lambda} \frac{d}{d x} x^{-\lambda}$, $\lambda>0$. We characterize the UMD property for a Banach space $\mathbb{B}$ by using $L^{p}((0, \infty), \mathbb{B})$-boundedness properties of $g$-functions defined by Bessel-Poisson semigroups. As a by-product, we prove that the fact that the imaginary power $\Delta_{\lambda}^{i \omega}, \omega \in \mathbb{R} \backslash\{0\}$, of the Bessel operator $\Delta_{\lambda}$ is bounded in $L^{p}((0, \infty), \mathbb{B})$, $1<p<\infty$, characterizes the UMD property for the Banach space $\mathbb{B}$. As applications of our results for square functions, we establish the boundedness in $L^{p}((0, \infty), \mathbb{B})$ of spectral multipliers $m\left(\Delta_{\lambda}\right)$ of Bessel operators defined by functions $m$ which are holomorphic in sectors $\Sigma_{\vartheta}$.
\end{abstract}

\section{Introduction}

Square functions (also called Littlewood-Paley $g$-functions) were considered in the works of Littlewood, Paley, Zygmund, and Marcinkiewicz during the decade of the 1930s (see [34], [55]). These functions were introduced to get new equivalent norms, for example, in $L^{p}$-spaces. By using these new equivalent norms the boundedness of some operators (e.g., multipliers) can be established.

Copyright 2016 by the Tusi Mathematical Research Group.

Received Apr. 10, 2015; Accepted Jul. 9, 2015.

${ }^{*}$ Corresponding author.

2010 Mathematics Subject Classification. Primary 42A25; Secondary 42B20, 43A15, 46B20, 46E40, 47D03.

Keywords. UMD space, square function, spectral multiplier, Bessel operator, $\gamma$-radonifying operator. 


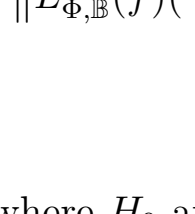

ANALYSIS

Banach J. Math. Anal. 10 (2016), no. 2, 338-384

http://dx.doi.org/10.1215/17358787-3495627

ISSN: $1735-8787$ (electronic)

http://projecteuclid.org/bjma

\title{
SQUARE FUNCTIONS AND SPECTRAL MULTIPLIERS FOR BESSEL OPERATORS IN UMD SPACES
}

\author{
JORGE J. BETANCOR, ${ }^{1}$ ALEJANDRO J. CASTRO,${ }^{2}$ and L. RODRÍGUEZ-MESA ${ }^{1 *}$ \\ Communicated by Q. Xu
}

\begin{abstract}
In this paper, we consider square functions (also called LittlewoodPaley $g$-functions) associated to Hankel convolutions acting on functions in the Bochner-Lebesgue space $L^{p}((0, \infty), \mathbb{B})$, where $\mathbb{B}$ is a UMD Banach space. As special cases, we study square functions defined by fractional derivatives of the Poisson semigroup for the Bessel operator $\Delta_{\lambda}=-x^{-\lambda} \frac{d}{d x} x^{2 \lambda} \frac{d}{d x} x^{-\lambda}$, $\lambda>0$. We characterize the UMD property for a Banach space $\mathbb{B}$ by using $L^{p}((0, \infty), \mathbb{B})$-boundedness properties of $g$-functions defined by Bessel-Poisson semigroups. As a by-product, we prove that the fact that the imaginary power $\Delta_{\lambda}^{i \omega}, \omega \in \mathbb{R} \backslash\{0\}$, of the Bessel operator $\Delta_{\lambda}$ is bounded in $L^{p}((0, \infty), \mathbb{B})$, $1<p<\infty$, characterizes the UMD property for the Banach space $\mathbb{B}$. As applications of our results for square functions, we establish the boundedness in $L^{p}((0, \infty), \mathbb{B})$ of spectral multipliers $m\left(\Delta_{\lambda}\right)$ of Bessel operators defined by functions $m$ which are holomorphic in sectors $\Sigma_{\vartheta}$.
\end{abstract}

\section{Introduction}

Square functions (also called Littlewood-Paley $g$-functions) were considered in the works of Littlewood, Paley, Zygmund, and Marcinkiewicz during the decade of the 1930s (see [34], [55]). These functions were introduced to get new equivalent norms, for example, in $L^{p}$-spaces. By using these new equivalent norms the boundedness of some operators (e.g., multipliers) can be established.

Copyright 2016 by the Tusi Mathematical Research Group.

Received Apr. 10, 2015; Accepted Jul. 9, 2015.

${ }^{*}$ Corresponding author.

2010 Mathematics Subject Classification. Primary 42A25; Secondary 42B20, 43A15, 46B20, 46E40, 47D03.

Keywords. UMD space, square function, spectral multiplier, Bessel operator, $\gamma$-radonifying operator. 


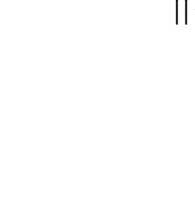

ANALYSIS

Banach J. Math. Anal. 10 (2016), no. 2, 338-384

http://dx.doi.org/10.1215/17358787-3495627

ISSN: $1735-8787$ (electronic)

http://projecteuclid.org/bjma

\title{
SQUARE FUNCTIONS AND SPECTRAL MULTIPLIERS FOR BESSEL OPERATORS IN UMD SPACES
}

\author{
JORGE J. BETANCOR, ${ }^{1}$ ALEJANDRO J. CASTRO,${ }^{2}$ and L. RODRÍGUEZ-MESA ${ }^{1 *}$ \\ Communicated by Q. Xu
}

\begin{abstract}
In this paper, we consider square functions (also called LittlewoodPaley $g$-functions) associated to Hankel convolutions acting on functions in the Bochner-Lebesgue space $L^{p}((0, \infty), \mathbb{B})$, where $\mathbb{B}$ is a UMD Banach space. As special cases, we study square functions defined by fractional derivatives of the Poisson semigroup for the Bessel operator $\Delta_{\lambda}=-x^{-\lambda} \frac{d}{d x} x^{2 \lambda} \frac{d}{d x} x^{-\lambda}$, $\lambda>0$. We characterize the UMD property for a Banach space $\mathbb{B}$ by using $L^{p}((0, \infty), \mathbb{B})$-boundedness properties of $g$-functions defined by Bessel-Poisson semigroups. As a by-product, we prove that the fact that the imaginary power $\Delta_{\lambda}^{i \omega}, \omega \in \mathbb{R} \backslash\{0\}$, of the Bessel operator $\Delta_{\lambda}$ is bounded in $L^{p}((0, \infty), \mathbb{B})$, $1<p<\infty$, characterizes the UMD property for the Banach space $\mathbb{B}$. As applications of our results for square functions, we establish the boundedness in $L^{p}((0, \infty), \mathbb{B})$ of spectral multipliers $m\left(\Delta_{\lambda}\right)$ of Bessel operators defined by functions $m$ which are holomorphic in sectors $\Sigma_{\vartheta}$.
\end{abstract}

\section{Introduction}

Square functions (also called Littlewood-Paley $g$-functions) were considered in the works of Littlewood, Paley, Zygmund, and Marcinkiewicz during the decade of the 1930s (see [34], [55]). These functions were introduced to get new equivalent norms, for example, in $L^{p}$-spaces. By using these new equivalent norms the boundedness of some operators (e.g., multipliers) can be established.

Copyright 2016 by the Tusi Mathematical Research Group.

Received Apr. 10, 2015; Accepted Jul. 9, 2015.

${ }^{*}$ Corresponding author.

2010 Mathematics Subject Classification. Primary 42A25; Secondary 42B20, 43A15, 46B20, 46E40, 47D03.

Keywords. UMD space, square function, spectral multiplier, Bessel operator, $\gamma$-radonifying operator. 


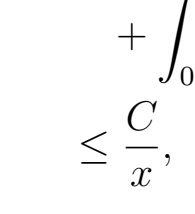

ANALYSIS

Banach J. Math. Anal. 10 (2016), no. 2, 338-384

http://dx.doi.org/10.1215/17358787-3495627

ISSN: $1735-8787$ (electronic)

http://projecteuclid.org/bjma

\title{
SQUARE FUNCTIONS AND SPECTRAL MULTIPLIERS FOR BESSEL OPERATORS IN UMD SPACES
}

\author{
JORGE J. BETANCOR, ${ }^{1}$ ALEJANDRO J. CASTRO,${ }^{2}$ and L. RODRÍGUEZ-MESA ${ }^{1 *}$ \\ Communicated by Q. Xu
}

\begin{abstract}
In this paper, we consider square functions (also called LittlewoodPaley $g$-functions) associated to Hankel convolutions acting on functions in the Bochner-Lebesgue space $L^{p}((0, \infty), \mathbb{B})$, where $\mathbb{B}$ is a UMD Banach space. As special cases, we study square functions defined by fractional derivatives of the Poisson semigroup for the Bessel operator $\Delta_{\lambda}=-x^{-\lambda} \frac{d}{d x} x^{2 \lambda} \frac{d}{d x} x^{-\lambda}$, $\lambda>0$. We characterize the UMD property for a Banach space $\mathbb{B}$ by using $L^{p}((0, \infty), \mathbb{B})$-boundedness properties of $g$-functions defined by Bessel-Poisson semigroups. As a by-product, we prove that the fact that the imaginary power $\Delta_{\lambda}^{i \omega}, \omega \in \mathbb{R} \backslash\{0\}$, of the Bessel operator $\Delta_{\lambda}$ is bounded in $L^{p}((0, \infty), \mathbb{B})$, $1<p<\infty$, characterizes the UMD property for the Banach space $\mathbb{B}$. As applications of our results for square functions, we establish the boundedness in $L^{p}((0, \infty), \mathbb{B})$ of spectral multipliers $m\left(\Delta_{\lambda}\right)$ of Bessel operators defined by functions $m$ which are holomorphic in sectors $\Sigma_{\vartheta}$.
\end{abstract}

\section{Introduction}

Square functions (also called Littlewood-Paley $g$-functions) were considered in the works of Littlewood, Paley, Zygmund, and Marcinkiewicz during the decade of the 1930s (see [34], [55]). These functions were introduced to get new equivalent norms, for example, in $L^{p}$-spaces. By using these new equivalent norms the boundedness of some operators (e.g., multipliers) can be established.

Copyright 2016 by the Tusi Mathematical Research Group.

Received Apr. 10, 2015; Accepted Jul. 9, 2015.

${ }^{*}$ Corresponding author.

2010 Mathematics Subject Classification. Primary 42A25; Secondary 42B20, 43A15, 46B20, 46E40, 47D03.

Keywords. UMD space, square function, spectral multiplier, Bessel operator, $\gamma$-radonifying operator. 


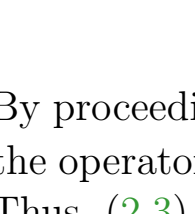

ANALYSIS

Banach J. Math. Anal. 10 (2016), no. 2, 338-384

http://dx.doi.org/10.1215/17358787-3495627

ISSN: $1735-8787$ (electronic)

http://projecteuclid.org/bjma

\title{
SQUARE FUNCTIONS AND SPECTRAL MULTIPLIERS FOR BESSEL OPERATORS IN UMD SPACES
}

\author{
JORGE J. BETANCOR, ${ }^{1}$ ALEJANDRO J. CASTRO,${ }^{2}$ and L. RODRÍGUEZ-MESA ${ }^{1 *}$ \\ Communicated by Q. Xu
}

\begin{abstract}
In this paper, we consider square functions (also called LittlewoodPaley $g$-functions) associated to Hankel convolutions acting on functions in the Bochner-Lebesgue space $L^{p}((0, \infty), \mathbb{B})$, where $\mathbb{B}$ is a UMD Banach space. As special cases, we study square functions defined by fractional derivatives of the Poisson semigroup for the Bessel operator $\Delta_{\lambda}=-x^{-\lambda} \frac{d}{d x} x^{2 \lambda} \frac{d}{d x} x^{-\lambda}$, $\lambda>0$. We characterize the UMD property for a Banach space $\mathbb{B}$ by using $L^{p}((0, \infty), \mathbb{B})$-boundedness properties of $g$-functions defined by Bessel-Poisson semigroups. As a by-product, we prove that the fact that the imaginary power $\Delta_{\lambda}^{i \omega}, \omega \in \mathbb{R} \backslash\{0\}$, of the Bessel operator $\Delta_{\lambda}$ is bounded in $L^{p}((0, \infty), \mathbb{B})$, $1<p<\infty$, characterizes the UMD property for the Banach space $\mathbb{B}$. As applications of our results for square functions, we establish the boundedness in $L^{p}((0, \infty), \mathbb{B})$ of spectral multipliers $m\left(\Delta_{\lambda}\right)$ of Bessel operators defined by functions $m$ which are holomorphic in sectors $\Sigma_{\vartheta}$.
\end{abstract}

\section{Introduction}

Square functions (also called Littlewood-Paley $g$-functions) were considered in the works of Littlewood, Paley, Zygmund, and Marcinkiewicz during the decade of the 1930s (see [34], [55]). These functions were introduced to get new equivalent norms, for example, in $L^{p}$-spaces. By using these new equivalent norms the boundedness of some operators (e.g., multipliers) can be established.

Copyright 2016 by the Tusi Mathematical Research Group.

Received Apr. 10, 2015; Accepted Jul. 9, 2015.

${ }^{*}$ Corresponding author.

2010 Mathematics Subject Classification. Primary 42A25; Secondary 42B20, 43A15, 46B20, 46E40, 47D03.

Keywords. UMD space, square function, spectral multiplier, Bessel operator, $\gamma$-radonifying operator. 


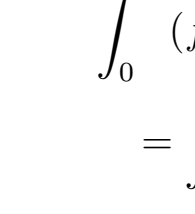

ANALYSIS

Banach J. Math. Anal. 10 (2016), no. 2, 338-384

http://dx.doi.org/10.1215/17358787-3495627

ISSN: $1735-8787$ (electronic)

http://projecteuclid.org/bjma

\title{
SQUARE FUNCTIONS AND SPECTRAL MULTIPLIERS FOR BESSEL OPERATORS IN UMD SPACES
}

\author{
JORGE J. BETANCOR, ${ }^{1}$ ALEJANDRO J. CASTRO,${ }^{2}$ and L. RODRÍGUEZ-MESA ${ }^{1 *}$ \\ Communicated by Q. Xu
}

\begin{abstract}
In this paper, we consider square functions (also called LittlewoodPaley $g$-functions) associated to Hankel convolutions acting on functions in the Bochner-Lebesgue space $L^{p}((0, \infty), \mathbb{B})$, where $\mathbb{B}$ is a UMD Banach space. As special cases, we study square functions defined by fractional derivatives of the Poisson semigroup for the Bessel operator $\Delta_{\lambda}=-x^{-\lambda} \frac{d}{d x} x^{2 \lambda} \frac{d}{d x} x^{-\lambda}$, $\lambda>0$. We characterize the UMD property for a Banach space $\mathbb{B}$ by using $L^{p}((0, \infty), \mathbb{B})$-boundedness properties of $g$-functions defined by Bessel-Poisson semigroups. As a by-product, we prove that the fact that the imaginary power $\Delta_{\lambda}^{i \omega}, \omega \in \mathbb{R} \backslash\{0\}$, of the Bessel operator $\Delta_{\lambda}$ is bounded in $L^{p}((0, \infty), \mathbb{B})$, $1<p<\infty$, characterizes the UMD property for the Banach space $\mathbb{B}$. As applications of our results for square functions, we establish the boundedness in $L^{p}((0, \infty), \mathbb{B})$ of spectral multipliers $m\left(\Delta_{\lambda}\right)$ of Bessel operators defined by functions $m$ which are holomorphic in sectors $\Sigma_{\vartheta}$.
\end{abstract}

\section{Introduction}

Square functions (also called Littlewood-Paley $g$-functions) were considered in the works of Littlewood, Paley, Zygmund, and Marcinkiewicz during the decade of the 1930s (see [34], [55]). These functions were introduced to get new equivalent norms, for example, in $L^{p}$-spaces. By using these new equivalent norms the boundedness of some operators (e.g., multipliers) can be established.

Copyright 2016 by the Tusi Mathematical Research Group.

Received Apr. 10, 2015; Accepted Jul. 9, 2015.

${ }^{*}$ Corresponding author.

2010 Mathematics Subject Classification. Primary 42A25; Secondary 42B20, 43A15, 46B20, 46E40, 47D03.

Keywords. UMD space, square function, spectral multiplier, Bessel operator, $\gamma$-radonifying operator. 


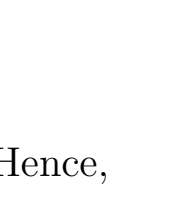

ANALYSIS

Banach J. Math. Anal. 10 (2016), no. 2, 338-384

http://dx.doi.org/10.1215/17358787-3495627

ISSN: $1735-8787$ (electronic)

http://projecteuclid.org/bjma

\title{
SQUARE FUNCTIONS AND SPECTRAL MULTIPLIERS FOR BESSEL OPERATORS IN UMD SPACES
}

\author{
JORGE J. BETANCOR, ${ }^{1}$ ALEJANDRO J. CASTRO,${ }^{2}$ and L. RODRÍGUEZ-MESA ${ }^{1 *}$ \\ Communicated by Q. Xu
}

\begin{abstract}
In this paper, we consider square functions (also called LittlewoodPaley $g$-functions) associated to Hankel convolutions acting on functions in the Bochner-Lebesgue space $L^{p}((0, \infty), \mathbb{B})$, where $\mathbb{B}$ is a UMD Banach space. As special cases, we study square functions defined by fractional derivatives of the Poisson semigroup for the Bessel operator $\Delta_{\lambda}=-x^{-\lambda} \frac{d}{d x} x^{2 \lambda} \frac{d}{d x} x^{-\lambda}$, $\lambda>0$. We characterize the UMD property for a Banach space $\mathbb{B}$ by using $L^{p}((0, \infty), \mathbb{B})$-boundedness properties of $g$-functions defined by Bessel-Poisson semigroups. As a by-product, we prove that the fact that the imaginary power $\Delta_{\lambda}^{i \omega}, \omega \in \mathbb{R} \backslash\{0\}$, of the Bessel operator $\Delta_{\lambda}$ is bounded in $L^{p}((0, \infty), \mathbb{B})$, $1<p<\infty$, characterizes the UMD property for the Banach space $\mathbb{B}$. As applications of our results for square functions, we establish the boundedness in $L^{p}((0, \infty), \mathbb{B})$ of spectral multipliers $m\left(\Delta_{\lambda}\right)$ of Bessel operators defined by functions $m$ which are holomorphic in sectors $\Sigma_{\vartheta}$.
\end{abstract}

\section{Introduction}

Square functions (also called Littlewood-Paley $g$-functions) were considered in the works of Littlewood, Paley, Zygmund, and Marcinkiewicz during the decade of the 1930s (see [34], [55]). These functions were introduced to get new equivalent norms, for example, in $L^{p}$-spaces. By using these new equivalent norms the boundedness of some operators (e.g., multipliers) can be established.

Copyright 2016 by the Tusi Mathematical Research Group.

Received Apr. 10, 2015; Accepted Jul. 9, 2015.

${ }^{*}$ Corresponding author.

2010 Mathematics Subject Classification. Primary 42A25; Secondary 42B20, 43A15, 46B20, 46E40, 47D03.

Keywords. UMD space, square function, spectral multiplier, Bessel operator, $\gamma$-radonifying operator. 


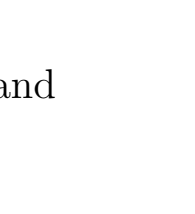

ANALYSIS

Banach J. Math. Anal. 10 (2016), no. 2, 338-384

http://dx.doi.org/10.1215/17358787-3495627

ISSN: $1735-8787$ (electronic)

http://projecteuclid.org/bjma

\title{
SQUARE FUNCTIONS AND SPECTRAL MULTIPLIERS FOR BESSEL OPERATORS IN UMD SPACES
}

\author{
JORGE J. BETANCOR, ${ }^{1}$ ALEJANDRO J. CASTRO,${ }^{2}$ and L. RODRÍGUEZ-MESA ${ }^{1 *}$ \\ Communicated by Q. Xu
}

\begin{abstract}
In this paper, we consider square functions (also called LittlewoodPaley $g$-functions) associated to Hankel convolutions acting on functions in the Bochner-Lebesgue space $L^{p}((0, \infty), \mathbb{B})$, where $\mathbb{B}$ is a UMD Banach space. As special cases, we study square functions defined by fractional derivatives of the Poisson semigroup for the Bessel operator $\Delta_{\lambda}=-x^{-\lambda} \frac{d}{d x} x^{2 \lambda} \frac{d}{d x} x^{-\lambda}$, $\lambda>0$. We characterize the UMD property for a Banach space $\mathbb{B}$ by using $L^{p}((0, \infty), \mathbb{B})$-boundedness properties of $g$-functions defined by Bessel-Poisson semigroups. As a by-product, we prove that the fact that the imaginary power $\Delta_{\lambda}^{i \omega}, \omega \in \mathbb{R} \backslash\{0\}$, of the Bessel operator $\Delta_{\lambda}$ is bounded in $L^{p}((0, \infty), \mathbb{B})$, $1<p<\infty$, characterizes the UMD property for the Banach space $\mathbb{B}$. As applications of our results for square functions, we establish the boundedness in $L^{p}((0, \infty), \mathbb{B})$ of spectral multipliers $m\left(\Delta_{\lambda}\right)$ of Bessel operators defined by functions $m$ which are holomorphic in sectors $\Sigma_{\vartheta}$.
\end{abstract}

\section{Introduction}

Square functions (also called Littlewood-Paley $g$-functions) were considered in the works of Littlewood, Paley, Zygmund, and Marcinkiewicz during the decade of the 1930s (see [34], [55]). These functions were introduced to get new equivalent norms, for example, in $L^{p}$-spaces. By using these new equivalent norms the boundedness of some operators (e.g., multipliers) can be established.

Copyright 2016 by the Tusi Mathematical Research Group.

Received Apr. 10, 2015; Accepted Jul. 9, 2015.

${ }^{*}$ Corresponding author.

2010 Mathematics Subject Classification. Primary 42A25; Secondary 42B20, 43A15, 46B20, 46E40, 47D03.

Keywords. UMD space, square function, spectral multiplier, Bessel operator, $\gamma$-radonifying operator. 


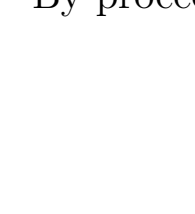

ANALYSIS

Banach J. Math. Anal. 10 (2016), no. 2, 338-384

http://dx.doi.org/10.1215/17358787-3495627

ISSN: $1735-8787$ (electronic)

http://projecteuclid.org/bjma

\title{
SQUARE FUNCTIONS AND SPECTRAL MULTIPLIERS FOR BESSEL OPERATORS IN UMD SPACES
}

\author{
JORGE J. BETANCOR, ${ }^{1}$ ALEJANDRO J. CASTRO,${ }^{2}$ and L. RODRÍGUEZ-MESA ${ }^{1 *}$ \\ Communicated by Q. Xu
}

\begin{abstract}
In this paper, we consider square functions (also called LittlewoodPaley $g$-functions) associated to Hankel convolutions acting on functions in the Bochner-Lebesgue space $L^{p}((0, \infty), \mathbb{B})$, where $\mathbb{B}$ is a UMD Banach space. As special cases, we study square functions defined by fractional derivatives of the Poisson semigroup for the Bessel operator $\Delta_{\lambda}=-x^{-\lambda} \frac{d}{d x} x^{2 \lambda} \frac{d}{d x} x^{-\lambda}$, $\lambda>0$. We characterize the UMD property for a Banach space $\mathbb{B}$ by using $L^{p}((0, \infty), \mathbb{B})$-boundedness properties of $g$-functions defined by Bessel-Poisson semigroups. As a by-product, we prove that the fact that the imaginary power $\Delta_{\lambda}^{i \omega}, \omega \in \mathbb{R} \backslash\{0\}$, of the Bessel operator $\Delta_{\lambda}$ is bounded in $L^{p}((0, \infty), \mathbb{B})$, $1<p<\infty$, characterizes the UMD property for the Banach space $\mathbb{B}$. As applications of our results for square functions, we establish the boundedness in $L^{p}((0, \infty), \mathbb{B})$ of spectral multipliers $m\left(\Delta_{\lambda}\right)$ of Bessel operators defined by functions $m$ which are holomorphic in sectors $\Sigma_{\vartheta}$.
\end{abstract}

\section{Introduction}

Square functions (also called Littlewood-Paley $g$-functions) were considered in the works of Littlewood, Paley, Zygmund, and Marcinkiewicz during the decade of the 1930s (see [34], [55]). These functions were introduced to get new equivalent norms, for example, in $L^{p}$-spaces. By using these new equivalent norms the boundedness of some operators (e.g., multipliers) can be established.

Copyright 2016 by the Tusi Mathematical Research Group.

Received Apr. 10, 2015; Accepted Jul. 9, 2015.

${ }^{*}$ Corresponding author.

2010 Mathematics Subject Classification. Primary 42A25; Secondary 42B20, 43A15, 46B20, 46E40, 47D03.

Keywords. UMD space, square function, spectral multiplier, Bessel operator, $\gamma$-radonifying operator. 


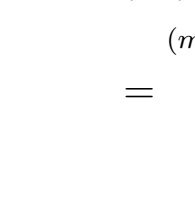

ANALYSIS

Banach J. Math. Anal. 10 (2016), no. 2, 338-384

http://dx.doi.org/10.1215/17358787-3495627

ISSN: $1735-8787$ (electronic)

http://projecteuclid.org/bjma

\title{
SQUARE FUNCTIONS AND SPECTRAL MULTIPLIERS FOR BESSEL OPERATORS IN UMD SPACES
}

\author{
JORGE J. BETANCOR, ${ }^{1}$ ALEJANDRO J. CASTRO,${ }^{2}$ and L. RODRÍGUEZ-MESA ${ }^{1 *}$ \\ Communicated by Q. Xu
}

\begin{abstract}
In this paper, we consider square functions (also called LittlewoodPaley $g$-functions) associated to Hankel convolutions acting on functions in the Bochner-Lebesgue space $L^{p}((0, \infty), \mathbb{B})$, where $\mathbb{B}$ is a UMD Banach space. As special cases, we study square functions defined by fractional derivatives of the Poisson semigroup for the Bessel operator $\Delta_{\lambda}=-x^{-\lambda} \frac{d}{d x} x^{2 \lambda} \frac{d}{d x} x^{-\lambda}$, $\lambda>0$. We characterize the UMD property for a Banach space $\mathbb{B}$ by using $L^{p}((0, \infty), \mathbb{B})$-boundedness properties of $g$-functions defined by Bessel-Poisson semigroups. As a by-product, we prove that the fact that the imaginary power $\Delta_{\lambda}^{i \omega}, \omega \in \mathbb{R} \backslash\{0\}$, of the Bessel operator $\Delta_{\lambda}$ is bounded in $L^{p}((0, \infty), \mathbb{B})$, $1<p<\infty$, characterizes the UMD property for the Banach space $\mathbb{B}$. As applications of our results for square functions, we establish the boundedness in $L^{p}((0, \infty), \mathbb{B})$ of spectral multipliers $m\left(\Delta_{\lambda}\right)$ of Bessel operators defined by functions $m$ which are holomorphic in sectors $\Sigma_{\vartheta}$.
\end{abstract}

\section{Introduction}

Square functions (also called Littlewood-Paley $g$-functions) were considered in the works of Littlewood, Paley, Zygmund, and Marcinkiewicz during the decade of the 1930s (see [34], [55]). These functions were introduced to get new equivalent norms, for example, in $L^{p}$-spaces. By using these new equivalent norms the boundedness of some operators (e.g., multipliers) can be established.

Copyright 2016 by the Tusi Mathematical Research Group.

Received Apr. 10, 2015; Accepted Jul. 9, 2015.

${ }^{*}$ Corresponding author.

2010 Mathematics Subject Classification. Primary 42A25; Secondary 42B20, 43A15, 46B20, 46E40, 47D03.

Keywords. UMD space, square function, spectral multiplier, Bessel operator, $\gamma$-radonifying operator. 


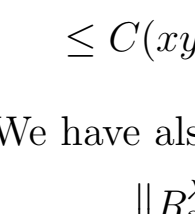

ANALYSIS

Banach J. Math. Anal. 10 (2016), no. 2, 338-384

http://dx.doi.org/10.1215/17358787-3495627

ISSN: $1735-8787$ (electronic)

http://projecteuclid.org/bjma

\title{
SQUARE FUNCTIONS AND SPECTRAL MULTIPLIERS FOR BESSEL OPERATORS IN UMD SPACES
}

\author{
JORGE J. BETANCOR, ${ }^{1}$ ALEJANDRO J. CASTRO,${ }^{2}$ and L. RODRÍGUEZ-MESA ${ }^{1 *}$ \\ Communicated by Q. Xu
}

\begin{abstract}
In this paper, we consider square functions (also called LittlewoodPaley $g$-functions) associated to Hankel convolutions acting on functions in the Bochner-Lebesgue space $L^{p}((0, \infty), \mathbb{B})$, where $\mathbb{B}$ is a UMD Banach space. As special cases, we study square functions defined by fractional derivatives of the Poisson semigroup for the Bessel operator $\Delta_{\lambda}=-x^{-\lambda} \frac{d}{d x} x^{2 \lambda} \frac{d}{d x} x^{-\lambda}$, $\lambda>0$. We characterize the UMD property for a Banach space $\mathbb{B}$ by using $L^{p}((0, \infty), \mathbb{B})$-boundedness properties of $g$-functions defined by Bessel-Poisson semigroups. As a by-product, we prove that the fact that the imaginary power $\Delta_{\lambda}^{i \omega}, \omega \in \mathbb{R} \backslash\{0\}$, of the Bessel operator $\Delta_{\lambda}$ is bounded in $L^{p}((0, \infty), \mathbb{B})$, $1<p<\infty$, characterizes the UMD property for the Banach space $\mathbb{B}$. As applications of our results for square functions, we establish the boundedness in $L^{p}((0, \infty), \mathbb{B})$ of spectral multipliers $m\left(\Delta_{\lambda}\right)$ of Bessel operators defined by functions $m$ which are holomorphic in sectors $\Sigma_{\vartheta}$.
\end{abstract}

\section{Introduction}

Square functions (also called Littlewood-Paley $g$-functions) were considered in the works of Littlewood, Paley, Zygmund, and Marcinkiewicz during the decade of the 1930s (see [34], [55]). These functions were introduced to get new equivalent norms, for example, in $L^{p}$-spaces. By using these new equivalent norms the boundedness of some operators (e.g., multipliers) can be established.

Copyright 2016 by the Tusi Mathematical Research Group.

Received Apr. 10, 2015; Accepted Jul. 9, 2015.

${ }^{*}$ Corresponding author.

2010 Mathematics Subject Classification. Primary 42A25; Secondary 42B20, 43A15, 46B20, 46E40, 47D03.

Keywords. UMD space, square function, spectral multiplier, Bessel operator, $\gamma$-radonifying operator. 


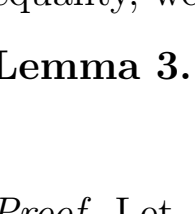

ANALYSIS

Banach J. Math. Anal. 10 (2016), no. 2, 338-384

http://dx.doi.org/10.1215/17358787-3495627

ISSN: $1735-8787$ (electronic)

http://projecteuclid.org/bjma

\title{
SQUARE FUNCTIONS AND SPECTRAL MULTIPLIERS FOR BESSEL OPERATORS IN UMD SPACES
}

\author{
JORGE J. BETANCOR, ${ }^{1}$ ALEJANDRO J. CASTRO,${ }^{2}$ and L. RODRÍGUEZ-MESA ${ }^{1 *}$ \\ Communicated by Q. Xu
}

\begin{abstract}
In this paper, we consider square functions (also called LittlewoodPaley $g$-functions) associated to Hankel convolutions acting on functions in the Bochner-Lebesgue space $L^{p}((0, \infty), \mathbb{B})$, where $\mathbb{B}$ is a UMD Banach space. As special cases, we study square functions defined by fractional derivatives of the Poisson semigroup for the Bessel operator $\Delta_{\lambda}=-x^{-\lambda} \frac{d}{d x} x^{2 \lambda} \frac{d}{d x} x^{-\lambda}$, $\lambda>0$. We characterize the UMD property for a Banach space $\mathbb{B}$ by using $L^{p}((0, \infty), \mathbb{B})$-boundedness properties of $g$-functions defined by Bessel-Poisson semigroups. As a by-product, we prove that the fact that the imaginary power $\Delta_{\lambda}^{i \omega}, \omega \in \mathbb{R} \backslash\{0\}$, of the Bessel operator $\Delta_{\lambda}$ is bounded in $L^{p}((0, \infty), \mathbb{B})$, $1<p<\infty$, characterizes the UMD property for the Banach space $\mathbb{B}$. As applications of our results for square functions, we establish the boundedness in $L^{p}((0, \infty), \mathbb{B})$ of spectral multipliers $m\left(\Delta_{\lambda}\right)$ of Bessel operators defined by functions $m$ which are holomorphic in sectors $\Sigma_{\vartheta}$.
\end{abstract}

\section{Introduction}

Square functions (also called Littlewood-Paley $g$-functions) were considered in the works of Littlewood, Paley, Zygmund, and Marcinkiewicz during the decade of the 1930s (see [34], [55]). These functions were introduced to get new equivalent norms, for example, in $L^{p}$-spaces. By using these new equivalent norms the boundedness of some operators (e.g., multipliers) can be established.

Copyright 2016 by the Tusi Mathematical Research Group.

Received Apr. 10, 2015; Accepted Jul. 9, 2015.

${ }^{*}$ Corresponding author.

2010 Mathematics Subject Classification. Primary 42A25; Secondary 42B20, 43A15, 46B20, 46E40, 47D03.

Keywords. UMD space, square function, spectral multiplier, Bessel operator, $\gamma$-radonifying operator. 


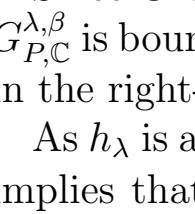

ANALYSIS

Banach J. Math. Anal. 10 (2016), no. 2, 338-384

http://dx.doi.org/10.1215/17358787-3495627

ISSN: $1735-8787$ (electronic)

http://projecteuclid.org/bjma

\title{
SQUARE FUNCTIONS AND SPECTRAL MULTIPLIERS FOR BESSEL OPERATORS IN UMD SPACES
}

\author{
JORGE J. BETANCOR, ${ }^{1}$ ALEJANDRO J. CASTRO,${ }^{2}$ and L. RODRÍGUEZ-MESA ${ }^{1 *}$ \\ Communicated by Q. Xu
}

\begin{abstract}
In this paper, we consider square functions (also called LittlewoodPaley $g$-functions) associated to Hankel convolutions acting on functions in the Bochner-Lebesgue space $L^{p}((0, \infty), \mathbb{B})$, where $\mathbb{B}$ is a UMD Banach space. As special cases, we study square functions defined by fractional derivatives of the Poisson semigroup for the Bessel operator $\Delta_{\lambda}=-x^{-\lambda} \frac{d}{d x} x^{2 \lambda} \frac{d}{d x} x^{-\lambda}$, $\lambda>0$. We characterize the UMD property for a Banach space $\mathbb{B}$ by using $L^{p}((0, \infty), \mathbb{B})$-boundedness properties of $g$-functions defined by Bessel-Poisson semigroups. As a by-product, we prove that the fact that the imaginary power $\Delta_{\lambda}^{i \omega}, \omega \in \mathbb{R} \backslash\{0\}$, of the Bessel operator $\Delta_{\lambda}$ is bounded in $L^{p}((0, \infty), \mathbb{B})$, $1<p<\infty$, characterizes the UMD property for the Banach space $\mathbb{B}$. As applications of our results for square functions, we establish the boundedness in $L^{p}((0, \infty), \mathbb{B})$ of spectral multipliers $m\left(\Delta_{\lambda}\right)$ of Bessel operators defined by functions $m$ which are holomorphic in sectors $\Sigma_{\vartheta}$.
\end{abstract}

\section{Introduction}

Square functions (also called Littlewood-Paley $g$-functions) were considered in the works of Littlewood, Paley, Zygmund, and Marcinkiewicz during the decade of the 1930s (see [34], [55]). These functions were introduced to get new equivalent norms, for example, in $L^{p}$-spaces. By using these new equivalent norms the boundedness of some operators (e.g., multipliers) can be established.

Copyright 2016 by the Tusi Mathematical Research Group.

Received Apr. 10, 2015; Accepted Jul. 9, 2015.

${ }^{*}$ Corresponding author.

2010 Mathematics Subject Classification. Primary 42A25; Secondary 42B20, 43A15, 46B20, 46E40, 47D03.

Keywords. UMD space, square function, spectral multiplier, Bessel operator, $\gamma$-radonifying operator. 


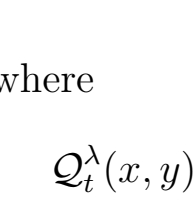

ANALYSIS

Banach J. Math. Anal. 10 (2016), no. 2, 338-384

http://dx.doi.org/10.1215/17358787-3495627

ISSN: $1735-8787$ (electronic)

http://projecteuclid.org/bjma

\title{
SQUARE FUNCTIONS AND SPECTRAL MULTIPLIERS FOR BESSEL OPERATORS IN UMD SPACES
}

\author{
JORGE J. BETANCOR, ${ }^{1}$ ALEJANDRO J. CASTRO,${ }^{2}$ and L. RODRÍGUEZ-MESA ${ }^{1 *}$ \\ Communicated by Q. Xu
}

\begin{abstract}
In this paper, we consider square functions (also called LittlewoodPaley $g$-functions) associated to Hankel convolutions acting on functions in the Bochner-Lebesgue space $L^{p}((0, \infty), \mathbb{B})$, where $\mathbb{B}$ is a UMD Banach space. As special cases, we study square functions defined by fractional derivatives of the Poisson semigroup for the Bessel operator $\Delta_{\lambda}=-x^{-\lambda} \frac{d}{d x} x^{2 \lambda} \frac{d}{d x} x^{-\lambda}$, $\lambda>0$. We characterize the UMD property for a Banach space $\mathbb{B}$ by using $L^{p}((0, \infty), \mathbb{B})$-boundedness properties of $g$-functions defined by Bessel-Poisson semigroups. As a by-product, we prove that the fact that the imaginary power $\Delta_{\lambda}^{i \omega}, \omega \in \mathbb{R} \backslash\{0\}$, of the Bessel operator $\Delta_{\lambda}$ is bounded in $L^{p}((0, \infty), \mathbb{B})$, $1<p<\infty$, characterizes the UMD property for the Banach space $\mathbb{B}$. As applications of our results for square functions, we establish the boundedness in $L^{p}((0, \infty), \mathbb{B})$ of spectral multipliers $m\left(\Delta_{\lambda}\right)$ of Bessel operators defined by functions $m$ which are holomorphic in sectors $\Sigma_{\vartheta}$.
\end{abstract}

\section{Introduction}

Square functions (also called Littlewood-Paley $g$-functions) were considered in the works of Littlewood, Paley, Zygmund, and Marcinkiewicz during the decade of the 1930s (see [34], [55]). These functions were introduced to get new equivalent norms, for example, in $L^{p}$-spaces. By using these new equivalent norms the boundedness of some operators (e.g., multipliers) can be established.

Copyright 2016 by the Tusi Mathematical Research Group.

Received Apr. 10, 2015; Accepted Jul. 9, 2015.

${ }^{*}$ Corresponding author.

2010 Mathematics Subject Classification. Primary 42A25; Secondary 42B20, 43A15, 46B20, 46E40, 47D03.

Keywords. UMD space, square function, spectral multiplier, Bessel operator, $\gamma$-radonifying operator. 


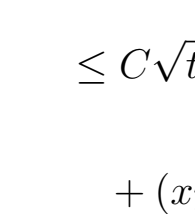

ANALYSIS

Banach J. Math. Anal. 10 (2016), no. 2, 338-384

http://dx.doi.org/10.1215/17358787-3495627

ISSN: $1735-8787$ (electronic)

http://projecteuclid.org/bjma

\title{
SQUARE FUNCTIONS AND SPECTRAL MULTIPLIERS FOR BESSEL OPERATORS IN UMD SPACES
}

\author{
JORGE J. BETANCOR, ${ }^{1}$ ALEJANDRO J. CASTRO,${ }^{2}$ and L. RODRÍGUEZ-MESA ${ }^{1 *}$ \\ Communicated by Q. Xu
}

\begin{abstract}
In this paper, we consider square functions (also called LittlewoodPaley $g$-functions) associated to Hankel convolutions acting on functions in the Bochner-Lebesgue space $L^{p}((0, \infty), \mathbb{B})$, where $\mathbb{B}$ is a UMD Banach space. As special cases, we study square functions defined by fractional derivatives of the Poisson semigroup for the Bessel operator $\Delta_{\lambda}=-x^{-\lambda} \frac{d}{d x} x^{2 \lambda} \frac{d}{d x} x^{-\lambda}$, $\lambda>0$. We characterize the UMD property for a Banach space $\mathbb{B}$ by using $L^{p}((0, \infty), \mathbb{B})$-boundedness properties of $g$-functions defined by Bessel-Poisson semigroups. As a by-product, we prove that the fact that the imaginary power $\Delta_{\lambda}^{i \omega}, \omega \in \mathbb{R} \backslash\{0\}$, of the Bessel operator $\Delta_{\lambda}$ is bounded in $L^{p}((0, \infty), \mathbb{B})$, $1<p<\infty$, characterizes the UMD property for the Banach space $\mathbb{B}$. As applications of our results for square functions, we establish the boundedness in $L^{p}((0, \infty), \mathbb{B})$ of spectral multipliers $m\left(\Delta_{\lambda}\right)$ of Bessel operators defined by functions $m$ which are holomorphic in sectors $\Sigma_{\vartheta}$.
\end{abstract}

\section{Introduction}

Square functions (also called Littlewood-Paley $g$-functions) were considered in the works of Littlewood, Paley, Zygmund, and Marcinkiewicz during the decade of the 1930s (see [34], [55]). These functions were introduced to get new equivalent norms, for example, in $L^{p}$-spaces. By using these new equivalent norms the boundedness of some operators (e.g., multipliers) can be established.

Copyright 2016 by the Tusi Mathematical Research Group.

Received Apr. 10, 2015; Accepted Jul. 9, 2015.

${ }^{*}$ Corresponding author.

2010 Mathematics Subject Classification. Primary 42A25; Secondary 42B20, 43A15, 46B20, 46E40, 47D03.

Keywords. UMD space, square function, spectral multiplier, Bessel operator, $\gamma$-radonifying operator. 


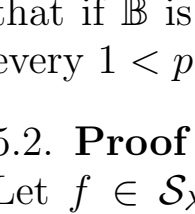

ANALYSIS

Banach J. Math. Anal. 10 (2016), no. 2, 338-384

http://dx.doi.org/10.1215/17358787-3495627

ISSN: $1735-8787$ (electronic)

http://projecteuclid.org/bjma

\title{
SQUARE FUNCTIONS AND SPECTRAL MULTIPLIERS FOR BESSEL OPERATORS IN UMD SPACES
}

\author{
JORGE J. BETANCOR, ${ }^{1}$ ALEJANDRO J. CASTRO,${ }^{2}$ and L. RODRÍGUEZ-MESA ${ }^{1 *}$ \\ Communicated by Q. Xu
}

\begin{abstract}
In this paper, we consider square functions (also called LittlewoodPaley $g$-functions) associated to Hankel convolutions acting on functions in the Bochner-Lebesgue space $L^{p}((0, \infty), \mathbb{B})$, where $\mathbb{B}$ is a UMD Banach space. As special cases, we study square functions defined by fractional derivatives of the Poisson semigroup for the Bessel operator $\Delta_{\lambda}=-x^{-\lambda} \frac{d}{d x} x^{2 \lambda} \frac{d}{d x} x^{-\lambda}$, $\lambda>0$. We characterize the UMD property for a Banach space $\mathbb{B}$ by using $L^{p}((0, \infty), \mathbb{B})$-boundedness properties of $g$-functions defined by Bessel-Poisson semigroups. As a by-product, we prove that the fact that the imaginary power $\Delta_{\lambda}^{i \omega}, \omega \in \mathbb{R} \backslash\{0\}$, of the Bessel operator $\Delta_{\lambda}$ is bounded in $L^{p}((0, \infty), \mathbb{B})$, $1<p<\infty$, characterizes the UMD property for the Banach space $\mathbb{B}$. As applications of our results for square functions, we establish the boundedness in $L^{p}((0, \infty), \mathbb{B})$ of spectral multipliers $m\left(\Delta_{\lambda}\right)$ of Bessel operators defined by functions $m$ which are holomorphic in sectors $\Sigma_{\vartheta}$.
\end{abstract}

\section{Introduction}

Square functions (also called Littlewood-Paley $g$-functions) were considered in the works of Littlewood, Paley, Zygmund, and Marcinkiewicz during the decade of the 1930s (see [34], [55]). These functions were introduced to get new equivalent norms, for example, in $L^{p}$-spaces. By using these new equivalent norms the boundedness of some operators (e.g., multipliers) can be established.

Copyright 2016 by the Tusi Mathematical Research Group.

Received Apr. 10, 2015; Accepted Jul. 9, 2015.

${ }^{*}$ Corresponding author.

2010 Mathematics Subject Classification. Primary 42A25; Secondary 42B20, 43A15, 46B20, 46E40, 47D03.

Keywords. UMD space, square function, spectral multiplier, Bessel operator, $\gamma$-radonifying operator. 


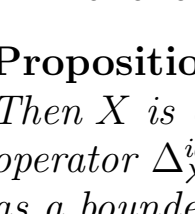

ANALYSIS

Banach J. Math. Anal. 10 (2016), no. 2, 338-384

http://dx.doi.org/10.1215/17358787-3495627

ISSN: $1735-8787$ (electronic)

http://projecteuclid.org/bjma

\title{
SQUARE FUNCTIONS AND SPECTRAL MULTIPLIERS FOR BESSEL OPERATORS IN UMD SPACES
}

\author{
JORGE J. BETANCOR, ${ }^{1}$ ALEJANDRO J. CASTRO,${ }^{2}$ and L. RODRÍGUEZ-MESA ${ }^{1 *}$ \\ Communicated by Q. Xu
}

\begin{abstract}
In this paper, we consider square functions (also called LittlewoodPaley $g$-functions) associated to Hankel convolutions acting on functions in the Bochner-Lebesgue space $L^{p}((0, \infty), \mathbb{B})$, where $\mathbb{B}$ is a UMD Banach space. As special cases, we study square functions defined by fractional derivatives of the Poisson semigroup for the Bessel operator $\Delta_{\lambda}=-x^{-\lambda} \frac{d}{d x} x^{2 \lambda} \frac{d}{d x} x^{-\lambda}$, $\lambda>0$. We characterize the UMD property for a Banach space $\mathbb{B}$ by using $L^{p}((0, \infty), \mathbb{B})$-boundedness properties of $g$-functions defined by Bessel-Poisson semigroups. As a by-product, we prove that the fact that the imaginary power $\Delta_{\lambda}^{i \omega}, \omega \in \mathbb{R} \backslash\{0\}$, of the Bessel operator $\Delta_{\lambda}$ is bounded in $L^{p}((0, \infty), \mathbb{B})$, $1<p<\infty$, characterizes the UMD property for the Banach space $\mathbb{B}$. As applications of our results for square functions, we establish the boundedness in $L^{p}((0, \infty), \mathbb{B})$ of spectral multipliers $m\left(\Delta_{\lambda}\right)$ of Bessel operators defined by functions $m$ which are holomorphic in sectors $\Sigma_{\vartheta}$.
\end{abstract}

\section{Introduction}

Square functions (also called Littlewood-Paley $g$-functions) were considered in the works of Littlewood, Paley, Zygmund, and Marcinkiewicz during the decade of the 1930s (see [34], [55]). These functions were introduced to get new equivalent norms, for example, in $L^{p}$-spaces. By using these new equivalent norms the boundedness of some operators (e.g., multipliers) can be established.

Copyright 2016 by the Tusi Mathematical Research Group.

Received Apr. 10, 2015; Accepted Jul. 9, 2015.

${ }^{*}$ Corresponding author.

2010 Mathematics Subject Classification. Primary 42A25; Secondary 42B20, 43A15, 46B20, 46E40, 47D03.

Keywords. UMD space, square function, spectral multiplier, Bessel operator, $\gamma$-radonifying operator. 


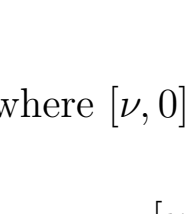

ANALYSIS

Banach J. Math. Anal. 10 (2016), no. 2, 338-384

http://dx.doi.org/10.1215/17358787-3495627

ISSN: $1735-8787$ (electronic)

http://projecteuclid.org/bjma

\title{
SQUARE FUNCTIONS AND SPECTRAL MULTIPLIERS FOR BESSEL OPERATORS IN UMD SPACES
}

\author{
JORGE J. BETANCOR, ${ }^{1}$ ALEJANDRO J. CASTRO,${ }^{2}$ and L. RODRÍGUEZ-MESA ${ }^{1 *}$ \\ Communicated by Q. Xu
}

\begin{abstract}
In this paper, we consider square functions (also called LittlewoodPaley $g$-functions) associated to Hankel convolutions acting on functions in the Bochner-Lebesgue space $L^{p}((0, \infty), \mathbb{B})$, where $\mathbb{B}$ is a UMD Banach space. As special cases, we study square functions defined by fractional derivatives of the Poisson semigroup for the Bessel operator $\Delta_{\lambda}=-x^{-\lambda} \frac{d}{d x} x^{2 \lambda} \frac{d}{d x} x^{-\lambda}$, $\lambda>0$. We characterize the UMD property for a Banach space $\mathbb{B}$ by using $L^{p}((0, \infty), \mathbb{B})$-boundedness properties of $g$-functions defined by Bessel-Poisson semigroups. As a by-product, we prove that the fact that the imaginary power $\Delta_{\lambda}^{i \omega}, \omega \in \mathbb{R} \backslash\{0\}$, of the Bessel operator $\Delta_{\lambda}$ is bounded in $L^{p}((0, \infty), \mathbb{B})$, $1<p<\infty$, characterizes the UMD property for the Banach space $\mathbb{B}$. As applications of our results for square functions, we establish the boundedness in $L^{p}((0, \infty), \mathbb{B})$ of spectral multipliers $m\left(\Delta_{\lambda}\right)$ of Bessel operators defined by functions $m$ which are holomorphic in sectors $\Sigma_{\vartheta}$.
\end{abstract}

\section{Introduction}

Square functions (also called Littlewood-Paley $g$-functions) were considered in the works of Littlewood, Paley, Zygmund, and Marcinkiewicz during the decade of the 1930s (see [34], [55]). These functions were introduced to get new equivalent norms, for example, in $L^{p}$-spaces. By using these new equivalent norms the boundedness of some operators (e.g., multipliers) can be established.

Copyright 2016 by the Tusi Mathematical Research Group.

Received Apr. 10, 2015; Accepted Jul. 9, 2015.

${ }^{*}$ Corresponding author.

2010 Mathematics Subject Classification. Primary 42A25; Secondary 42B20, 43A15, 46B20, 46E40, 47D03.

Keywords. UMD space, square function, spectral multiplier, Bessel operator, $\gamma$-radonifying operator. 


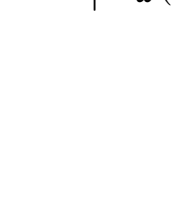

ANALYSIS

Banach J. Math. Anal. 10 (2016), no. 2, 338-384

http://dx.doi.org/10.1215/17358787-3495627

ISSN: $1735-8787$ (electronic)

http://projecteuclid.org/bjma

\title{
SQUARE FUNCTIONS AND SPECTRAL MULTIPLIERS FOR BESSEL OPERATORS IN UMD SPACES
}

\author{
JORGE J. BETANCOR, ${ }^{1}$ ALEJANDRO J. CASTRO,${ }^{2}$ and L. RODRÍGUEZ-MESA ${ }^{1 *}$ \\ Communicated by Q. Xu
}

\begin{abstract}
In this paper, we consider square functions (also called LittlewoodPaley $g$-functions) associated to Hankel convolutions acting on functions in the Bochner-Lebesgue space $L^{p}((0, \infty), \mathbb{B})$, where $\mathbb{B}$ is a UMD Banach space. As special cases, we study square functions defined by fractional derivatives of the Poisson semigroup for the Bessel operator $\Delta_{\lambda}=-x^{-\lambda} \frac{d}{d x} x^{2 \lambda} \frac{d}{d x} x^{-\lambda}$, $\lambda>0$. We characterize the UMD property for a Banach space $\mathbb{B}$ by using $L^{p}((0, \infty), \mathbb{B})$-boundedness properties of $g$-functions defined by Bessel-Poisson semigroups. As a by-product, we prove that the fact that the imaginary power $\Delta_{\lambda}^{i \omega}, \omega \in \mathbb{R} \backslash\{0\}$, of the Bessel operator $\Delta_{\lambda}$ is bounded in $L^{p}((0, \infty), \mathbb{B})$, $1<p<\infty$, characterizes the UMD property for the Banach space $\mathbb{B}$. As applications of our results for square functions, we establish the boundedness in $L^{p}((0, \infty), \mathbb{B})$ of spectral multipliers $m\left(\Delta_{\lambda}\right)$ of Bessel operators defined by functions $m$ which are holomorphic in sectors $\Sigma_{\vartheta}$.
\end{abstract}

\section{Introduction}

Square functions (also called Littlewood-Paley $g$-functions) were considered in the works of Littlewood, Paley, Zygmund, and Marcinkiewicz during the decade of the 1930s (see [34], [55]). These functions were introduced to get new equivalent norms, for example, in $L^{p}$-spaces. By using these new equivalent norms the boundedness of some operators (e.g., multipliers) can be established.

Copyright 2016 by the Tusi Mathematical Research Group.

Received Apr. 10, 2015; Accepted Jul. 9, 2015.

${ }^{*}$ Corresponding author.

2010 Mathematics Subject Classification. Primary 42A25; Secondary 42B20, 43A15, 46B20, 46E40, 47D03.

Keywords. UMD space, square function, spectral multiplier, Bessel operator, $\gamma$-radonifying operator. 


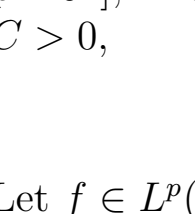

ANALYSIS

Banach J. Math. Anal. 10 (2016), no. 2, 338-384

http://dx.doi.org/10.1215/17358787-3495627

ISSN: $1735-8787$ (electronic)

http://projecteuclid.org/bjma

\title{
SQUARE FUNCTIONS AND SPECTRAL MULTIPLIERS FOR BESSEL OPERATORS IN UMD SPACES
}

\author{
JORGE J. BETANCOR, ${ }^{1}$ ALEJANDRO J. CASTRO,${ }^{2}$ and L. RODRÍGUEZ-MESA ${ }^{1 *}$ \\ Communicated by Q. Xu
}

\begin{abstract}
In this paper, we consider square functions (also called LittlewoodPaley $g$-functions) associated to Hankel convolutions acting on functions in the Bochner-Lebesgue space $L^{p}((0, \infty), \mathbb{B})$, where $\mathbb{B}$ is a UMD Banach space. As special cases, we study square functions defined by fractional derivatives of the Poisson semigroup for the Bessel operator $\Delta_{\lambda}=-x^{-\lambda} \frac{d}{d x} x^{2 \lambda} \frac{d}{d x} x^{-\lambda}$, $\lambda>0$. We characterize the UMD property for a Banach space $\mathbb{B}$ by using $L^{p}((0, \infty), \mathbb{B})$-boundedness properties of $g$-functions defined by Bessel-Poisson semigroups. As a by-product, we prove that the fact that the imaginary power $\Delta_{\lambda}^{i \omega}, \omega \in \mathbb{R} \backslash\{0\}$, of the Bessel operator $\Delta_{\lambda}$ is bounded in $L^{p}((0, \infty), \mathbb{B})$, $1<p<\infty$, characterizes the UMD property for the Banach space $\mathbb{B}$. As applications of our results for square functions, we establish the boundedness in $L^{p}((0, \infty), \mathbb{B})$ of spectral multipliers $m\left(\Delta_{\lambda}\right)$ of Bessel operators defined by functions $m$ which are holomorphic in sectors $\Sigma_{\vartheta}$.
\end{abstract}

\section{Introduction}

Square functions (also called Littlewood-Paley $g$-functions) were considered in the works of Littlewood, Paley, Zygmund, and Marcinkiewicz during the decade of the 1930s (see [34], [55]). These functions were introduced to get new equivalent norms, for example, in $L^{p}$-spaces. By using these new equivalent norms the boundedness of some operators (e.g., multipliers) can be established.

Copyright 2016 by the Tusi Mathematical Research Group.

Received Apr. 10, 2015; Accepted Jul. 9, 2015.

${ }^{*}$ Corresponding author.

2010 Mathematics Subject Classification. Primary 42A25; Secondary 42B20, 43A15, 46B20, 46E40, 47D03.

Keywords. UMD space, square function, spectral multiplier, Bessel operator, $\gamma$-radonifying operator. 


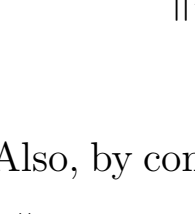

ANALYSIS

Banach J. Math. Anal. 10 (2016), no. 2, 338-384

http://dx.doi.org/10.1215/17358787-3495627

ISSN: $1735-8787$ (electronic)

http://projecteuclid.org/bjma

\title{
SQUARE FUNCTIONS AND SPECTRAL MULTIPLIERS FOR BESSEL OPERATORS IN UMD SPACES
}

\author{
JORGE J. BETANCOR, ${ }^{1}$ ALEJANDRO J. CASTRO,${ }^{2}$ and L. RODRÍGUEZ-MESA ${ }^{1 *}$ \\ Communicated by Q. Xu
}

\begin{abstract}
In this paper, we consider square functions (also called LittlewoodPaley $g$-functions) associated to Hankel convolutions acting on functions in the Bochner-Lebesgue space $L^{p}((0, \infty), \mathbb{B})$, where $\mathbb{B}$ is a UMD Banach space. As special cases, we study square functions defined by fractional derivatives of the Poisson semigroup for the Bessel operator $\Delta_{\lambda}=-x^{-\lambda} \frac{d}{d x} x^{2 \lambda} \frac{d}{d x} x^{-\lambda}$, $\lambda>0$. We characterize the UMD property for a Banach space $\mathbb{B}$ by using $L^{p}((0, \infty), \mathbb{B})$-boundedness properties of $g$-functions defined by Bessel-Poisson semigroups. As a by-product, we prove that the fact that the imaginary power $\Delta_{\lambda}^{i \omega}, \omega \in \mathbb{R} \backslash\{0\}$, of the Bessel operator $\Delta_{\lambda}$ is bounded in $L^{p}((0, \infty), \mathbb{B})$, $1<p<\infty$, characterizes the UMD property for the Banach space $\mathbb{B}$. As applications of our results for square functions, we establish the boundedness in $L^{p}((0, \infty), \mathbb{B})$ of spectral multipliers $m\left(\Delta_{\lambda}\right)$ of Bessel operators defined by functions $m$ which are holomorphic in sectors $\Sigma_{\vartheta}$.
\end{abstract}

\section{Introduction}

Square functions (also called Littlewood-Paley $g$-functions) were considered in the works of Littlewood, Paley, Zygmund, and Marcinkiewicz during the decade of the 1930s (see [34], [55]). These functions were introduced to get new equivalent norms, for example, in $L^{p}$-spaces. By using these new equivalent norms the boundedness of some operators (e.g., multipliers) can be established.

Copyright 2016 by the Tusi Mathematical Research Group.

Received Apr. 10, 2015; Accepted Jul. 9, 2015.

${ }^{*}$ Corresponding author.

2010 Mathematics Subject Classification. Primary 42A25; Secondary 42B20, 43A15, 46B20, 46E40, 47D03.

Keywords. UMD space, square function, spectral multiplier, Bessel operator, $\gamma$-radonifying operator. 


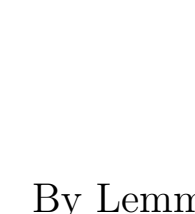

ANALYSIS

Banach J. Math. Anal. 10 (2016), no. 2, 338-384

http://dx.doi.org/10.1215/17358787-3495627

ISSN: $1735-8787$ (electronic)

http://projecteuclid.org/bjma

\title{
SQUARE FUNCTIONS AND SPECTRAL MULTIPLIERS FOR BESSEL OPERATORS IN UMD SPACES
}

\author{
JORGE J. BETANCOR, ${ }^{1}$ ALEJANDRO J. CASTRO,${ }^{2}$ and L. RODRÍGUEZ-MESA ${ }^{1 *}$ \\ Communicated by Q. Xu
}

\begin{abstract}
In this paper, we consider square functions (also called LittlewoodPaley $g$-functions) associated to Hankel convolutions acting on functions in the Bochner-Lebesgue space $L^{p}((0, \infty), \mathbb{B})$, where $\mathbb{B}$ is a UMD Banach space. As special cases, we study square functions defined by fractional derivatives of the Poisson semigroup for the Bessel operator $\Delta_{\lambda}=-x^{-\lambda} \frac{d}{d x} x^{2 \lambda} \frac{d}{d x} x^{-\lambda}$, $\lambda>0$. We characterize the UMD property for a Banach space $\mathbb{B}$ by using $L^{p}((0, \infty), \mathbb{B})$-boundedness properties of $g$-functions defined by Bessel-Poisson semigroups. As a by-product, we prove that the fact that the imaginary power $\Delta_{\lambda}^{i \omega}, \omega \in \mathbb{R} \backslash\{0\}$, of the Bessel operator $\Delta_{\lambda}$ is bounded in $L^{p}((0, \infty), \mathbb{B})$, $1<p<\infty$, characterizes the UMD property for the Banach space $\mathbb{B}$. As applications of our results for square functions, we establish the boundedness in $L^{p}((0, \infty), \mathbb{B})$ of spectral multipliers $m\left(\Delta_{\lambda}\right)$ of Bessel operators defined by functions $m$ which are holomorphic in sectors $\Sigma_{\vartheta}$.
\end{abstract}

\section{Introduction}

Square functions (also called Littlewood-Paley $g$-functions) were considered in the works of Littlewood, Paley, Zygmund, and Marcinkiewicz during the decade of the 1930s (see [34], [55]). These functions were introduced to get new equivalent norms, for example, in $L^{p}$-spaces. By using these new equivalent norms the boundedness of some operators (e.g., multipliers) can be established.

Copyright 2016 by the Tusi Mathematical Research Group.

Received Apr. 10, 2015; Accepted Jul. 9, 2015.

${ }^{*}$ Corresponding author.

2010 Mathematics Subject Classification. Primary 42A25; Secondary 42B20, 43A15, 46B20, 46E40, 47D03.

Keywords. UMD space, square function, spectral multiplier, Bessel operator, $\gamma$-radonifying operator. 


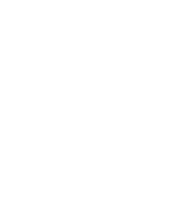

ANALYSIS

Banach J. Math. Anal. 10 (2016), no. 2, 338-384

http://dx.doi.org/10.1215/17358787-3495627

ISSN: $1735-8787$ (electronic)

http://projecteuclid.org/bjma

\title{
SQUARE FUNCTIONS AND SPECTRAL MULTIPLIERS FOR BESSEL OPERATORS IN UMD SPACES
}

\author{
JORGE J. BETANCOR, ${ }^{1}$ ALEJANDRO J. CASTRO,${ }^{2}$ and L. RODRÍGUEZ-MESA ${ }^{1 *}$ \\ Communicated by Q. Xu
}

\begin{abstract}
In this paper, we consider square functions (also called LittlewoodPaley $g$-functions) associated to Hankel convolutions acting on functions in the Bochner-Lebesgue space $L^{p}((0, \infty), \mathbb{B})$, where $\mathbb{B}$ is a UMD Banach space. As special cases, we study square functions defined by fractional derivatives of the Poisson semigroup for the Bessel operator $\Delta_{\lambda}=-x^{-\lambda} \frac{d}{d x} x^{2 \lambda} \frac{d}{d x} x^{-\lambda}$, $\lambda>0$. We characterize the UMD property for a Banach space $\mathbb{B}$ by using $L^{p}((0, \infty), \mathbb{B})$-boundedness properties of $g$-functions defined by Bessel-Poisson semigroups. As a by-product, we prove that the fact that the imaginary power $\Delta_{\lambda}^{i \omega}, \omega \in \mathbb{R} \backslash\{0\}$, of the Bessel operator $\Delta_{\lambda}$ is bounded in $L^{p}((0, \infty), \mathbb{B})$, $1<p<\infty$, characterizes the UMD property for the Banach space $\mathbb{B}$. As applications of our results for square functions, we establish the boundedness in $L^{p}((0, \infty), \mathbb{B})$ of spectral multipliers $m\left(\Delta_{\lambda}\right)$ of Bessel operators defined by functions $m$ which are holomorphic in sectors $\Sigma_{\vartheta}$.
\end{abstract}

\section{Introduction}

Square functions (also called Littlewood-Paley $g$-functions) were considered in the works of Littlewood, Paley, Zygmund, and Marcinkiewicz during the decade of the 1930s (see [34], [55]). These functions were introduced to get new equivalent norms, for example, in $L^{p}$-spaces. By using these new equivalent norms the boundedness of some operators (e.g., multipliers) can be established.

Copyright 2016 by the Tusi Mathematical Research Group.

Received Apr. 10, 2015; Accepted Jul. 9, 2015.

${ }^{*}$ Corresponding author.

2010 Mathematics Subject Classification. Primary 42A25; Secondary 42B20, 43A15, 46B20, 46E40, 47D03.

Keywords. UMD space, square function, spectral multiplier, Bessel operator, $\gamma$-radonifying operator. 


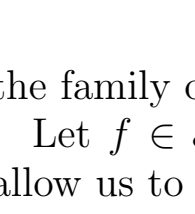

ANALYSIS

Banach J. Math. Anal. 10 (2016), no. 2, 338-384

http://dx.doi.org/10.1215/17358787-3495627

ISSN: $1735-8787$ (electronic)

http://projecteuclid.org/bjma

\title{
SQUARE FUNCTIONS AND SPECTRAL MULTIPLIERS FOR BESSEL OPERATORS IN UMD SPACES
}

\author{
JORGE J. BETANCOR, ${ }^{1}$ ALEJANDRO J. CASTRO,${ }^{2}$ and L. RODRÍGUEZ-MESA ${ }^{1 *}$ \\ Communicated by Q. Xu
}

\begin{abstract}
In this paper, we consider square functions (also called LittlewoodPaley $g$-functions) associated to Hankel convolutions acting on functions in the Bochner-Lebesgue space $L^{p}((0, \infty), \mathbb{B})$, where $\mathbb{B}$ is a UMD Banach space. As special cases, we study square functions defined by fractional derivatives of the Poisson semigroup for the Bessel operator $\Delta_{\lambda}=-x^{-\lambda} \frac{d}{d x} x^{2 \lambda} \frac{d}{d x} x^{-\lambda}$, $\lambda>0$. We characterize the UMD property for a Banach space $\mathbb{B}$ by using $L^{p}((0, \infty), \mathbb{B})$-boundedness properties of $g$-functions defined by Bessel-Poisson semigroups. As a by-product, we prove that the fact that the imaginary power $\Delta_{\lambda}^{i \omega}, \omega \in \mathbb{R} \backslash\{0\}$, of the Bessel operator $\Delta_{\lambda}$ is bounded in $L^{p}((0, \infty), \mathbb{B})$, $1<p<\infty$, characterizes the UMD property for the Banach space $\mathbb{B}$. As applications of our results for square functions, we establish the boundedness in $L^{p}((0, \infty), \mathbb{B})$ of spectral multipliers $m\left(\Delta_{\lambda}\right)$ of Bessel operators defined by functions $m$ which are holomorphic in sectors $\Sigma_{\vartheta}$.
\end{abstract}

\section{Introduction}

Square functions (also called Littlewood-Paley $g$-functions) were considered in the works of Littlewood, Paley, Zygmund, and Marcinkiewicz during the decade of the 1930s (see [34], [55]). These functions were introduced to get new equivalent norms, for example, in $L^{p}$-spaces. By using these new equivalent norms the boundedness of some operators (e.g., multipliers) can be established.

Copyright 2016 by the Tusi Mathematical Research Group.

Received Apr. 10, 2015; Accepted Jul. 9, 2015.

${ }^{*}$ Corresponding author.

2010 Mathematics Subject Classification. Primary 42A25; Secondary 42B20, 43A15, 46B20, 46E40, 47D03.

Keywords. UMD space, square function, spectral multiplier, Bessel operator, $\gamma$-radonifying operator. 


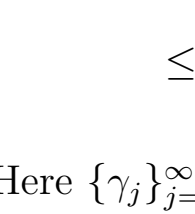

ANALYSIS

Banach J. Math. Anal. 10 (2016), no. 2, 338-384

http://dx.doi.org/10.1215/17358787-3495627

ISSN: $1735-8787$ (electronic)

http://projecteuclid.org/bjma

\title{
SQUARE FUNCTIONS AND SPECTRAL MULTIPLIERS FOR BESSEL OPERATORS IN UMD SPACES
}

\author{
JORGE J. BETANCOR, ${ }^{1}$ ALEJANDRO J. CASTRO,${ }^{2}$ and L. RODRÍGUEZ-MESA ${ }^{1 *}$ \\ Communicated by Q. Xu
}

\begin{abstract}
In this paper, we consider square functions (also called LittlewoodPaley $g$-functions) associated to Hankel convolutions acting on functions in the Bochner-Lebesgue space $L^{p}((0, \infty), \mathbb{B})$, where $\mathbb{B}$ is a UMD Banach space. As special cases, we study square functions defined by fractional derivatives of the Poisson semigroup for the Bessel operator $\Delta_{\lambda}=-x^{-\lambda} \frac{d}{d x} x^{2 \lambda} \frac{d}{d x} x^{-\lambda}$, $\lambda>0$. We characterize the UMD property for a Banach space $\mathbb{B}$ by using $L^{p}((0, \infty), \mathbb{B})$-boundedness properties of $g$-functions defined by Bessel-Poisson semigroups. As a by-product, we prove that the fact that the imaginary power $\Delta_{\lambda}^{i \omega}, \omega \in \mathbb{R} \backslash\{0\}$, of the Bessel operator $\Delta_{\lambda}$ is bounded in $L^{p}((0, \infty), \mathbb{B})$, $1<p<\infty$, characterizes the UMD property for the Banach space $\mathbb{B}$. As applications of our results for square functions, we establish the boundedness in $L^{p}((0, \infty), \mathbb{B})$ of spectral multipliers $m\left(\Delta_{\lambda}\right)$ of Bessel operators defined by functions $m$ which are holomorphic in sectors $\Sigma_{\vartheta}$.
\end{abstract}

\section{Introduction}

Square functions (also called Littlewood-Paley $g$-functions) were considered in the works of Littlewood, Paley, Zygmund, and Marcinkiewicz during the decade of the 1930s (see [34], [55]). These functions were introduced to get new equivalent norms, for example, in $L^{p}$-spaces. By using these new equivalent norms the boundedness of some operators (e.g., multipliers) can be established.

Copyright 2016 by the Tusi Mathematical Research Group.

Received Apr. 10, 2015; Accepted Jul. 9, 2015.

${ }^{*}$ Corresponding author.

2010 Mathematics Subject Classification. Primary 42A25; Secondary 42B20, 43A15, 46B20, 46E40, 47D03.

Keywords. UMD space, square function, spectral multiplier, Bessel operator, $\gamma$-radonifying operator. 


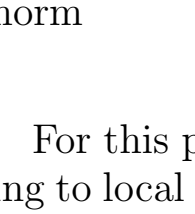

ANALYSIS

Banach J. Math. Anal. 10 (2016), no. 2, 338-384

http://dx.doi.org/10.1215/17358787-3495627

ISSN: $1735-8787$ (electronic)

http://projecteuclid.org/bjma

\title{
SQUARE FUNCTIONS AND SPECTRAL MULTIPLIERS FOR BESSEL OPERATORS IN UMD SPACES
}

\author{
JORGE J. BETANCOR, ${ }^{1}$ ALEJANDRO J. CASTRO,${ }^{2}$ and L. RODRÍGUEZ-MESA ${ }^{1 *}$ \\ Communicated by Q. Xu
}

\begin{abstract}
In this paper, we consider square functions (also called LittlewoodPaley $g$-functions) associated to Hankel convolutions acting on functions in the Bochner-Lebesgue space $L^{p}((0, \infty), \mathbb{B})$, where $\mathbb{B}$ is a UMD Banach space. As special cases, we study square functions defined by fractional derivatives of the Poisson semigroup for the Bessel operator $\Delta_{\lambda}=-x^{-\lambda} \frac{d}{d x} x^{2 \lambda} \frac{d}{d x} x^{-\lambda}$, $\lambda>0$. We characterize the UMD property for a Banach space $\mathbb{B}$ by using $L^{p}((0, \infty), \mathbb{B})$-boundedness properties of $g$-functions defined by Bessel-Poisson semigroups. As a by-product, we prove that the fact that the imaginary power $\Delta_{\lambda}^{i \omega}, \omega \in \mathbb{R} \backslash\{0\}$, of the Bessel operator $\Delta_{\lambda}$ is bounded in $L^{p}((0, \infty), \mathbb{B})$, $1<p<\infty$, characterizes the UMD property for the Banach space $\mathbb{B}$. As applications of our results for square functions, we establish the boundedness in $L^{p}((0, \infty), \mathbb{B})$ of spectral multipliers $m\left(\Delta_{\lambda}\right)$ of Bessel operators defined by functions $m$ which are holomorphic in sectors $\Sigma_{\vartheta}$.
\end{abstract}

\section{Introduction}

Square functions (also called Littlewood-Paley $g$-functions) were considered in the works of Littlewood, Paley, Zygmund, and Marcinkiewicz during the decade of the 1930s (see [34], [55]). These functions were introduced to get new equivalent norms, for example, in $L^{p}$-spaces. By using these new equivalent norms the boundedness of some operators (e.g., multipliers) can be established.

Copyright 2016 by the Tusi Mathematical Research Group.

Received Apr. 10, 2015; Accepted Jul. 9, 2015.

${ }^{*}$ Corresponding author.

2010 Mathematics Subject Classification. Primary 42A25; Secondary 42B20, 43A15, 46B20, 46E40, 47D03.

Keywords. UMD space, square function, spectral multiplier, Bessel operator, $\gamma$-radonifying operator. 


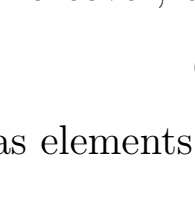

ANALYSIS

Banach J. Math. Anal. 10 (2016), no. 2, 338-384

http://dx.doi.org/10.1215/17358787-3495627

ISSN: $1735-8787$ (electronic)

http://projecteuclid.org/bjma

\title{
SQUARE FUNCTIONS AND SPECTRAL MULTIPLIERS FOR BESSEL OPERATORS IN UMD SPACES
}

\author{
JORGE J. BETANCOR, ${ }^{1}$ ALEJANDRO J. CASTRO,${ }^{2}$ and L. RODRÍGUEZ-MESA ${ }^{1 *}$ \\ Communicated by Q. Xu
}

\begin{abstract}
In this paper, we consider square functions (also called LittlewoodPaley $g$-functions) associated to Hankel convolutions acting on functions in the Bochner-Lebesgue space $L^{p}((0, \infty), \mathbb{B})$, where $\mathbb{B}$ is a UMD Banach space. As special cases, we study square functions defined by fractional derivatives of the Poisson semigroup for the Bessel operator $\Delta_{\lambda}=-x^{-\lambda} \frac{d}{d x} x^{2 \lambda} \frac{d}{d x} x^{-\lambda}$, $\lambda>0$. We characterize the UMD property for a Banach space $\mathbb{B}$ by using $L^{p}((0, \infty), \mathbb{B})$-boundedness properties of $g$-functions defined by Bessel-Poisson semigroups. As a by-product, we prove that the fact that the imaginary power $\Delta_{\lambda}^{i \omega}, \omega \in \mathbb{R} \backslash\{0\}$, of the Bessel operator $\Delta_{\lambda}$ is bounded in $L^{p}((0, \infty), \mathbb{B})$, $1<p<\infty$, characterizes the UMD property for the Banach space $\mathbb{B}$. As applications of our results for square functions, we establish the boundedness in $L^{p}((0, \infty), \mathbb{B})$ of spectral multipliers $m\left(\Delta_{\lambda}\right)$ of Bessel operators defined by functions $m$ which are holomorphic in sectors $\Sigma_{\vartheta}$.
\end{abstract}

\section{Introduction}

Square functions (also called Littlewood-Paley $g$-functions) were considered in the works of Littlewood, Paley, Zygmund, and Marcinkiewicz during the decade of the 1930s (see [34], [55]). These functions were introduced to get new equivalent norms, for example, in $L^{p}$-spaces. By using these new equivalent norms the boundedness of some operators (e.g., multipliers) can be established.

Copyright 2016 by the Tusi Mathematical Research Group.

Received Apr. 10, 2015; Accepted Jul. 9, 2015.

${ }^{*}$ Corresponding author.

2010 Mathematics Subject Classification. Primary 42A25; Secondary 42B20, 43A15, 46B20, 46E40, 47D03.

Keywords. UMD space, square function, spectral multiplier, Bessel operator, $\gamma$-radonifying operator. 


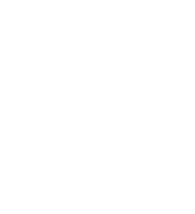

ANALYSIS

Banach J. Math. Anal. 10 (2016), no. 2, 338-384

http://dx.doi.org/10.1215/17358787-3495627

ISSN: $1735-8787$ (electronic)

http://projecteuclid.org/bjma

\title{
SQUARE FUNCTIONS AND SPECTRAL MULTIPLIERS FOR BESSEL OPERATORS IN UMD SPACES
}

\author{
JORGE J. BETANCOR, ${ }^{1}$ ALEJANDRO J. CASTRO,${ }^{2}$ and L. RODRÍGUEZ-MESA ${ }^{1 *}$ \\ Communicated by Q. Xu
}

\begin{abstract}
In this paper, we consider square functions (also called LittlewoodPaley $g$-functions) associated to Hankel convolutions acting on functions in the Bochner-Lebesgue space $L^{p}((0, \infty), \mathbb{B})$, where $\mathbb{B}$ is a UMD Banach space. As special cases, we study square functions defined by fractional derivatives of the Poisson semigroup for the Bessel operator $\Delta_{\lambda}=-x^{-\lambda} \frac{d}{d x} x^{2 \lambda} \frac{d}{d x} x^{-\lambda}$, $\lambda>0$. We characterize the UMD property for a Banach space $\mathbb{B}$ by using $L^{p}((0, \infty), \mathbb{B})$-boundedness properties of $g$-functions defined by Bessel-Poisson semigroups. As a by-product, we prove that the fact that the imaginary power $\Delta_{\lambda}^{i \omega}, \omega \in \mathbb{R} \backslash\{0\}$, of the Bessel operator $\Delta_{\lambda}$ is bounded in $L^{p}((0, \infty), \mathbb{B})$, $1<p<\infty$, characterizes the UMD property for the Banach space $\mathbb{B}$. As applications of our results for square functions, we establish the boundedness in $L^{p}((0, \infty), \mathbb{B})$ of spectral multipliers $m\left(\Delta_{\lambda}\right)$ of Bessel operators defined by functions $m$ which are holomorphic in sectors $\Sigma_{\vartheta}$.
\end{abstract}

\section{Introduction}

Square functions (also called Littlewood-Paley $g$-functions) were considered in the works of Littlewood, Paley, Zygmund, and Marcinkiewicz during the decade of the 1930s (see [34], [55]). These functions were introduced to get new equivalent norms, for example, in $L^{p}$-spaces. By using these new equivalent norms the boundedness of some operators (e.g., multipliers) can be established.

Copyright 2016 by the Tusi Mathematical Research Group.

Received Apr. 10, 2015; Accepted Jul. 9, 2015.

${ }^{*}$ Corresponding author.

2010 Mathematics Subject Classification. Primary 42A25; Secondary 42B20, 43A15, 46B20, 46E40, 47D03.

Keywords. UMD space, square function, spectral multiplier, Bessel operator, $\gamma$-radonifying operator. 


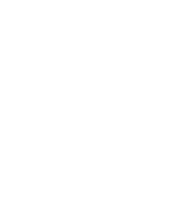

ANALYSIS

Banach J. Math. Anal. 10 (2016), no. 2, 338-384

http://dx.doi.org/10.1215/17358787-3495627

ISSN: $1735-8787$ (electronic)

http://projecteuclid.org/bjma

\title{
SQUARE FUNCTIONS AND SPECTRAL MULTIPLIERS FOR BESSEL OPERATORS IN UMD SPACES
}

\author{
JORGE J. BETANCOR, ${ }^{1}$ ALEJANDRO J. CASTRO,${ }^{2}$ and L. RODRÍGUEZ-MESA ${ }^{1 *}$ \\ Communicated by Q. Xu
}

\begin{abstract}
In this paper, we consider square functions (also called LittlewoodPaley $g$-functions) associated to Hankel convolutions acting on functions in the Bochner-Lebesgue space $L^{p}((0, \infty), \mathbb{B})$, where $\mathbb{B}$ is a UMD Banach space. As special cases, we study square functions defined by fractional derivatives of the Poisson semigroup for the Bessel operator $\Delta_{\lambda}=-x^{-\lambda} \frac{d}{d x} x^{2 \lambda} \frac{d}{d x} x^{-\lambda}$, $\lambda>0$. We characterize the UMD property for a Banach space $\mathbb{B}$ by using $L^{p}((0, \infty), \mathbb{B})$-boundedness properties of $g$-functions defined by Bessel-Poisson semigroups. As a by-product, we prove that the fact that the imaginary power $\Delta_{\lambda}^{i \omega}, \omega \in \mathbb{R} \backslash\{0\}$, of the Bessel operator $\Delta_{\lambda}$ is bounded in $L^{p}((0, \infty), \mathbb{B})$, $1<p<\infty$, characterizes the UMD property for the Banach space $\mathbb{B}$. As applications of our results for square functions, we establish the boundedness in $L^{p}((0, \infty), \mathbb{B})$ of spectral multipliers $m\left(\Delta_{\lambda}\right)$ of Bessel operators defined by functions $m$ which are holomorphic in sectors $\Sigma_{\vartheta}$.
\end{abstract}

\section{Introduction}

Square functions (also called Littlewood-Paley $g$-functions) were considered in the works of Littlewood, Paley, Zygmund, and Marcinkiewicz during the decade of the 1930s (see [34], [55]). These functions were introduced to get new equivalent norms, for example, in $L^{p}$-spaces. By using these new equivalent norms the boundedness of some operators (e.g., multipliers) can be established.

Copyright 2016 by the Tusi Mathematical Research Group.

Received Apr. 10, 2015; Accepted Jul. 9, 2015.

${ }^{*}$ Corresponding author.

2010 Mathematics Subject Classification. Primary 42A25; Secondary 42B20, 43A15, 46B20, 46E40, 47D03.

Keywords. UMD space, square function, spectral multiplier, Bessel operator, $\gamma$-radonifying operator. 


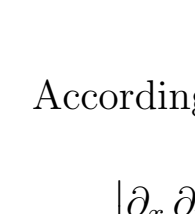

ANALYSIS

Banach J. Math. Anal. 10 (2016), no. 2, 338-384

http://dx.doi.org/10.1215/17358787-3495627

ISSN: $1735-8787$ (electronic)

http://projecteuclid.org/bjma

\title{
SQUARE FUNCTIONS AND SPECTRAL MULTIPLIERS FOR BESSEL OPERATORS IN UMD SPACES
}

\author{
JORGE J. BETANCOR, ${ }^{1}$ ALEJANDRO J. CASTRO,${ }^{2}$ and L. RODRÍGUEZ-MESA ${ }^{1 *}$ \\ Communicated by Q. Xu
}

\begin{abstract}
In this paper, we consider square functions (also called LittlewoodPaley $g$-functions) associated to Hankel convolutions acting on functions in the Bochner-Lebesgue space $L^{p}((0, \infty), \mathbb{B})$, where $\mathbb{B}$ is a UMD Banach space. As special cases, we study square functions defined by fractional derivatives of the Poisson semigroup for the Bessel operator $\Delta_{\lambda}=-x^{-\lambda} \frac{d}{d x} x^{2 \lambda} \frac{d}{d x} x^{-\lambda}$, $\lambda>0$. We characterize the UMD property for a Banach space $\mathbb{B}$ by using $L^{p}((0, \infty), \mathbb{B})$-boundedness properties of $g$-functions defined by Bessel-Poisson semigroups. As a by-product, we prove that the fact that the imaginary power $\Delta_{\lambda}^{i \omega}, \omega \in \mathbb{R} \backslash\{0\}$, of the Bessel operator $\Delta_{\lambda}$ is bounded in $L^{p}((0, \infty), \mathbb{B})$, $1<p<\infty$, characterizes the UMD property for the Banach space $\mathbb{B}$. As applications of our results for square functions, we establish the boundedness in $L^{p}((0, \infty), \mathbb{B})$ of spectral multipliers $m\left(\Delta_{\lambda}\right)$ of Bessel operators defined by functions $m$ which are holomorphic in sectors $\Sigma_{\vartheta}$.
\end{abstract}

\section{Introduction}

Square functions (also called Littlewood-Paley $g$-functions) were considered in the works of Littlewood, Paley, Zygmund, and Marcinkiewicz during the decade of the 1930s (see [34], [55]). These functions were introduced to get new equivalent norms, for example, in $L^{p}$-spaces. By using these new equivalent norms the boundedness of some operators (e.g., multipliers) can be established.

Copyright 2016 by the Tusi Mathematical Research Group.

Received Apr. 10, 2015; Accepted Jul. 9, 2015.

${ }^{*}$ Corresponding author.

2010 Mathematics Subject Classification. Primary 42A25; Secondary 42B20, 43A15, 46B20, 46E40, 47D03.

Keywords. UMD space, square function, spectral multiplier, Bessel operator, $\gamma$-radonifying operator. 


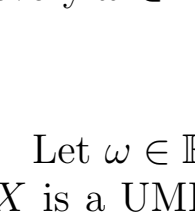

ANALYSIS

Banach J. Math. Anal. 10 (2016), no. 2, 338-384

http://dx.doi.org/10.1215/17358787-3495627

ISSN: $1735-8787$ (electronic)

http://projecteuclid.org/bjma

\title{
SQUARE FUNCTIONS AND SPECTRAL MULTIPLIERS FOR BESSEL OPERATORS IN UMD SPACES
}

\author{
JORGE J. BETANCOR, ${ }^{1}$ ALEJANDRO J. CASTRO,${ }^{2}$ and L. RODRÍGUEZ-MESA ${ }^{1 *}$ \\ Communicated by Q. Xu
}

\begin{abstract}
In this paper, we consider square functions (also called LittlewoodPaley $g$-functions) associated to Hankel convolutions acting on functions in the Bochner-Lebesgue space $L^{p}((0, \infty), \mathbb{B})$, where $\mathbb{B}$ is a UMD Banach space. As special cases, we study square functions defined by fractional derivatives of the Poisson semigroup for the Bessel operator $\Delta_{\lambda}=-x^{-\lambda} \frac{d}{d x} x^{2 \lambda} \frac{d}{d x} x^{-\lambda}$, $\lambda>0$. We characterize the UMD property for a Banach space $\mathbb{B}$ by using $L^{p}((0, \infty), \mathbb{B})$-boundedness properties of $g$-functions defined by Bessel-Poisson semigroups. As a by-product, we prove that the fact that the imaginary power $\Delta_{\lambda}^{i \omega}, \omega \in \mathbb{R} \backslash\{0\}$, of the Bessel operator $\Delta_{\lambda}$ is bounded in $L^{p}((0, \infty), \mathbb{B})$, $1<p<\infty$, characterizes the UMD property for the Banach space $\mathbb{B}$. As applications of our results for square functions, we establish the boundedness in $L^{p}((0, \infty), \mathbb{B})$ of spectral multipliers $m\left(\Delta_{\lambda}\right)$ of Bessel operators defined by functions $m$ which are holomorphic in sectors $\Sigma_{\vartheta}$.
\end{abstract}

\section{Introduction}

Square functions (also called Littlewood-Paley $g$-functions) were considered in the works of Littlewood, Paley, Zygmund, and Marcinkiewicz during the decade of the 1930s (see [34], [55]). These functions were introduced to get new equivalent norms, for example, in $L^{p}$-spaces. By using these new equivalent norms the boundedness of some operators (e.g., multipliers) can be established.

Copyright 2016 by the Tusi Mathematical Research Group.

Received Apr. 10, 2015; Accepted Jul. 9, 2015.

${ }^{*}$ Corresponding author.

2010 Mathematics Subject Classification. Primary 42A25; Secondary 42B20, 43A15, 46B20, 46E40, 47D03.

Keywords. UMD space, square function, spectral multiplier, Bessel operator, $\gamma$-radonifying operator. 


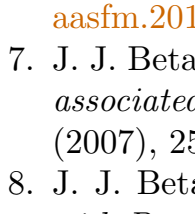

ANALYSIS

Banach J. Math. Anal. 10 (2016), no. 2, 338-384

http://dx.doi.org/10.1215/17358787-3495627

ISSN: $1735-8787$ (electronic)

http://projecteuclid.org/bjma

\title{
SQUARE FUNCTIONS AND SPECTRAL MULTIPLIERS FOR BESSEL OPERATORS IN UMD SPACES
}

\author{
JORGE J. BETANCOR, ${ }^{1}$ ALEJANDRO J. CASTRO,${ }^{2}$ and L. RODRÍGUEZ-MESA ${ }^{1 *}$ \\ Communicated by Q. Xu
}

\begin{abstract}
In this paper, we consider square functions (also called LittlewoodPaley $g$-functions) associated to Hankel convolutions acting on functions in the Bochner-Lebesgue space $L^{p}((0, \infty), \mathbb{B})$, where $\mathbb{B}$ is a UMD Banach space. As special cases, we study square functions defined by fractional derivatives of the Poisson semigroup for the Bessel operator $\Delta_{\lambda}=-x^{-\lambda} \frac{d}{d x} x^{2 \lambda} \frac{d}{d x} x^{-\lambda}$, $\lambda>0$. We characterize the UMD property for a Banach space $\mathbb{B}$ by using $L^{p}((0, \infty), \mathbb{B})$-boundedness properties of $g$-functions defined by Bessel-Poisson semigroups. As a by-product, we prove that the fact that the imaginary power $\Delta_{\lambda}^{i \omega}, \omega \in \mathbb{R} \backslash\{0\}$, of the Bessel operator $\Delta_{\lambda}$ is bounded in $L^{p}((0, \infty), \mathbb{B})$, $1<p<\infty$, characterizes the UMD property for the Banach space $\mathbb{B}$. As applications of our results for square functions, we establish the boundedness in $L^{p}((0, \infty), \mathbb{B})$ of spectral multipliers $m\left(\Delta_{\lambda}\right)$ of Bessel operators defined by functions $m$ which are holomorphic in sectors $\Sigma_{\vartheta}$.
\end{abstract}

\section{Introduction}

Square functions (also called Littlewood-Paley $g$-functions) were considered in the works of Littlewood, Paley, Zygmund, and Marcinkiewicz during the decade of the 1930s (see [34], [55]). These functions were introduced to get new equivalent norms, for example, in $L^{p}$-spaces. By using these new equivalent norms the boundedness of some operators (e.g., multipliers) can be established.

Copyright 2016 by the Tusi Mathematical Research Group.

Received Apr. 10, 2015; Accepted Jul. 9, 2015.

${ }^{*}$ Corresponding author.

2010 Mathematics Subject Classification. Primary 42A25; Secondary 42B20, 43A15, 46B20, 46E40, 47D03.

Keywords. UMD space, square function, spectral multiplier, Bessel operator, $\gamma$-radonifying operator. 


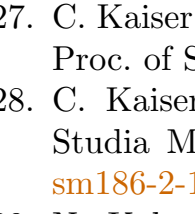

ANALYSIS

Banach J. Math. Anal. 10 (2016), no. 2, 338-384

http://dx.doi.org/10.1215/17358787-3495627

ISSN: $1735-8787$ (electronic)

http://projecteuclid.org/bjma

\title{
SQUARE FUNCTIONS AND SPECTRAL MULTIPLIERS FOR BESSEL OPERATORS IN UMD SPACES
}

\author{
JORGE J. BETANCOR, ${ }^{1}$ ALEJANDRO J. CASTRO,${ }^{2}$ and L. RODRÍGUEZ-MESA ${ }^{1 *}$ \\ Communicated by Q. Xu
}

\begin{abstract}
In this paper, we consider square functions (also called LittlewoodPaley $g$-functions) associated to Hankel convolutions acting on functions in the Bochner-Lebesgue space $L^{p}((0, \infty), \mathbb{B})$, where $\mathbb{B}$ is a UMD Banach space. As special cases, we study square functions defined by fractional derivatives of the Poisson semigroup for the Bessel operator $\Delta_{\lambda}=-x^{-\lambda} \frac{d}{d x} x^{2 \lambda} \frac{d}{d x} x^{-\lambda}$, $\lambda>0$. We characterize the UMD property for a Banach space $\mathbb{B}$ by using $L^{p}((0, \infty), \mathbb{B})$-boundedness properties of $g$-functions defined by Bessel-Poisson semigroups. As a by-product, we prove that the fact that the imaginary power $\Delta_{\lambda}^{i \omega}, \omega \in \mathbb{R} \backslash\{0\}$, of the Bessel operator $\Delta_{\lambda}$ is bounded in $L^{p}((0, \infty), \mathbb{B})$, $1<p<\infty$, characterizes the UMD property for the Banach space $\mathbb{B}$. As applications of our results for square functions, we establish the boundedness in $L^{p}((0, \infty), \mathbb{B})$ of spectral multipliers $m\left(\Delta_{\lambda}\right)$ of Bessel operators defined by functions $m$ which are holomorphic in sectors $\Sigma_{\vartheta}$.
\end{abstract}

\section{Introduction}

Square functions (also called Littlewood-Paley $g$-functions) were considered in the works of Littlewood, Paley, Zygmund, and Marcinkiewicz during the decade of the 1930s (see [34], [55]). These functions were introduced to get new equivalent norms, for example, in $L^{p}$-spaces. By using these new equivalent norms the boundedness of some operators (e.g., multipliers) can be established.

Copyright 2016 by the Tusi Mathematical Research Group.

Received Apr. 10, 2015; Accepted Jul. 9, 2015.

${ }^{*}$ Corresponding author.

2010 Mathematics Subject Classification. Primary 42A25; Secondary 42B20, 43A15, 46B20, 46E40, 47D03.

Keywords. UMD space, square function, spectral multiplier, Bessel operator, $\gamma$-radonifying operator. 


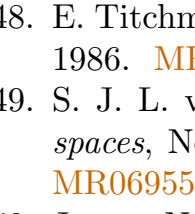

ANALYSIS

Banach J. Math. Anal. 10 (2016), no. 2, 338-384

http://dx.doi.org/10.1215/17358787-3495627

ISSN: $1735-8787$ (electronic)

http://projecteuclid.org/bjma

\title{
SQUARE FUNCTIONS AND SPECTRAL MULTIPLIERS FOR BESSEL OPERATORS IN UMD SPACES
}

\author{
JORGE J. BETANCOR, ${ }^{1}$ ALEJANDRO J. CASTRO,${ }^{2}$ and L. RODRÍGUEZ-MESA ${ }^{1 *}$ \\ Communicated by Q. Xu
}

\begin{abstract}
In this paper, we consider square functions (also called LittlewoodPaley $g$-functions) associated to Hankel convolutions acting on functions in the Bochner-Lebesgue space $L^{p}((0, \infty), \mathbb{B})$, where $\mathbb{B}$ is a UMD Banach space. As special cases, we study square functions defined by fractional derivatives of the Poisson semigroup for the Bessel operator $\Delta_{\lambda}=-x^{-\lambda} \frac{d}{d x} x^{2 \lambda} \frac{d}{d x} x^{-\lambda}$, $\lambda>0$. We characterize the UMD property for a Banach space $\mathbb{B}$ by using $L^{p}((0, \infty), \mathbb{B})$-boundedness properties of $g$-functions defined by Bessel-Poisson semigroups. As a by-product, we prove that the fact that the imaginary power $\Delta_{\lambda}^{i \omega}, \omega \in \mathbb{R} \backslash\{0\}$, of the Bessel operator $\Delta_{\lambda}$ is bounded in $L^{p}((0, \infty), \mathbb{B})$, $1<p<\infty$, characterizes the UMD property for the Banach space $\mathbb{B}$. As applications of our results for square functions, we establish the boundedness in $L^{p}((0, \infty), \mathbb{B})$ of spectral multipliers $m\left(\Delta_{\lambda}\right)$ of Bessel operators defined by functions $m$ which are holomorphic in sectors $\Sigma_{\vartheta}$.
\end{abstract}

\section{Introduction}

Square functions (also called Littlewood-Paley $g$-functions) were considered in the works of Littlewood, Paley, Zygmund, and Marcinkiewicz during the decade of the 1930s (see [34], [55]). These functions were introduced to get new equivalent norms, for example, in $L^{p}$-spaces. By using these new equivalent norms the boundedness of some operators (e.g., multipliers) can be established.

Copyright 2016 by the Tusi Mathematical Research Group.

Received Apr. 10, 2015; Accepted Jul. 9, 2015.

${ }^{*}$ Corresponding author.

2010 Mathematics Subject Classification. Primary 42A25; Secondary 42B20, 43A15, 46B20, 46E40, 47D03.

Keywords. UMD space, square function, spectral multiplier, Bessel operator, $\gamma$-radonifying operator. 